\title{
Signal and error in mass change inferences from GRACE: the case of Antarctica
}

\author{
Martin Horwath* $†$ and Reinhard Dietrich \\ Technische Universität Dresden, Institut für Planetare Geodäsie, D-01062 Dresden, Germany. E-mail: martin.horwath@legos.obs-mip.fr
}

Accepted 2009 February 2. Received 2009 February 2; in original form 2008 June 10

\begin{abstract}
SUMMAR Y
This paper examines mass changes from Gravity Recovery and Climate Experiment (GRACE) monthly spherical harmonic gravity field solutions, focusing on the application to the Antarctic ice sheet. Regional integration approaches are investigated and compared with an alternative technique of fitting predefined mass change patterns. The main thrust of this new analysis is to better define error sources and error bounds on mass changes of the Antarctic ice sheet. Toward this purpose, we examine the release-4 GRACE products by the GeoForschungsZentrum Potsdam. Although errors related to the correction for glacial isostatic adjustment are significant, both leakage and GRACE error effects are also important. In particular, the details of how mass change interior to the Antarctic continent is weighted and scaled is crucially linked to the end-result mass trend solution. Accounting for error correlations in the GRACE monthly solutions, it is shown that the corresponding errors in Antarctic mass changes are about two times larger than predicted by uncorrelated error propagation. Our ice mass trend estimate for the grounded ice sheet in the period from $08 / 2002$ to $01 / 2008$ is $(-109 \pm 48) \mathrm{Gt} \mathrm{yr}^{-1}$, equivalent to a mean eustatic sea level rise of $(+0.30 \pm 0.13) \mathrm{mm} \mathrm{yr}^{-1}$. We also provide estimates for 16 individual drainage basins. Mass losses concentrate in the coastal regions of West Antarctica and on the Antarctic Peninsula with together $(-105 \pm 35) \mathrm{Gt} \mathrm{yr}^{-1}$. The Amundsen Sea Sector alone contributes $(-60 \pm 8) \mathrm{Gt} \mathrm{yr}^{-1}$. Although these findings agree with independent observations, GRACE trends deduced from a short time-series come with the cautioning footnote that the strength of interannual variations remains undetermined. We finally discuss directions of methodological improvements.
\end{abstract}

Key words: Satellite geodesy; Sea level change; Time variable gravity; Global change from geodesy; Glaciology; Antarctica.

\section{INTRODUCTION}

The Gravity Recovery and Climate Experiment (GRACE) satellite mission has proven an extremely useful tool for observing mass transports in the Earth system (Tapley et al. 2004; Schmidt et al. 2006). The processes of interest include changes of the Antarctic ice sheet. Those changes involve a large range of temporal and spatial scales and include, in particular, variations in ice flow. Since these processes remain poorly understood, the Antarctic ice mass balance both in the last few decades and in the future is uncertain even in sign, which adds a crucial uncertainty to global sea level predictions (Solomon et al. 2007).

The common way of making geophysical inferences from GRACE data comprises two distinct steps: First, GRACE process-

\footnotetext{
*Now at: LEGOS (UMR $5566 \mathrm{CNES/CNRS/IRD/UPS),} \mathrm{18,} \mathrm{Avenue} \mathrm{E.}$ Belin, 31401 Toulouse Cedex 9, France.

†Birth name: Wiehl.
}

ing centres such as the GeoForschungsZentrum Potsdam (GFZ), the Center for Space Research at the University of Texas at Austin (UTCSR), or the Centre National d'Etudes Spatiales / Groupe de Recherche de Géodésie Spatiale Toulouse (CNES/GRGS) process the GRACE observations to produce global solutions of the timevariable gravity field, typically monthly solutions. They are solved in a spherical harmonic $(\mathrm{SH})$ representation and are provided to the users. In a second step, the users analyse these monthly solutions to estimate geophysical mass changes.

We note that there exist attractive alternatives, for example, the mascon approach (Luthcke et al. 2006, 2007; Han et al. 2005). The topic of this paper, however, is the two-step approach with $\mathrm{SH}$ gravity field solutions.

Following this two-step approach, a number of studies have inferred Antarctic ice mass changes from GRACE (Chen et al. 2006, 2008; Horwath \& Dietrich 2006; Ramillien et al. 2006; Velicogna \& Wahr 2006a; Llubes et al. 2007; Sasgen et al. 2007). Although they use data from the same mission, their results differ considerably. Compare, for example, the trend estimate of $(-139 \pm 73) \mathrm{Gt} \mathrm{yr}^{-1}$ for the period $04 / 2002-08 / 2005$ by Velicogna \& Wahr (2006a) 
and the estimate of $(-40 \pm 36) \mathrm{Gt} \mathrm{yr}^{-1}$ for $07 / 2002-03 / 2005$ by Ramillien et al. (2006).

Interannual ice mass variations are one cause of differences. However, even with restriction to identical time intervals, very different results can arise from different releases of monthly solutions (e.g. Sasgen et al. 2007) or from different methods of analysing one set of monthly solutions.

One root cause of differences may be traced to discrepant ways of handling glacial isostatic adjustment (GIA) signals and separating these from ice mass signals. However, other differences of methods are also important. It is desirable to understand the error mechanisms of the different methods to resolve the differences between results and to advance the methodologies.

This paper aims to thoroughly investigate methods and errors of mass change inferences from GRACE monthly solutions given in the SH representation. Our area of application is Antarctica and our numerical results use the release- 4 monthly solutions by GFZ, yet the methods and insights are adaptable to other regions and to other monthly solution series. After providing a general framework of estimation methods and their inherent errors in Section 2, we justify our practical realization of mass change estimates in Section 3. We place special emphasis on error assessments (Section 4) and include intermediate and auxiliary results to isolate different sources for discrepant results. The extended analysis aids in disclosing current and future challenges for the SH GRACE solution method. We provide new estimates of ice mass changes of the Antarctic ice sheet and its large drainage basins (Section 5) and, finally, conclude with a discussion of methodological improvements (Section 6).

\section{METHODS OF MASS CHANGE ESTIMATES FROM GRACE MONTHLY SOLUTIONS}

\subsection{General framework of mass change estimates}

Following the approach of Wahr et al. (1998) and Swenson \& Wahr (2002) mass variations are modelled as surface density variations $\Delta \kappa(\Omega, t)$ in a spherical layer of radius $a$ ( $a$ is the semi major axis, $\Omega$ and $t$ are the spherical position and the time, the unit of $\Delta \kappa$ is mass per surface area). The mass change within a given region, or integration region, is expressed as the following integral over the unit sphere:

$\Delta m(t)=\iint_{\Omega} \vartheta(\Omega) \Delta \kappa(\Omega, t) a^{2} \mathrm{~d} \Omega$,

where the region function $\vartheta(\Omega)$ is defined as 1 inside the region and 0 outside. Typically, the considered mass variations are residuals with respect to a background model or set of models, and our task is to estimate mass changes according to (1) from GRACE monthly gravity field solutions.

These monthly solutions are given in the SH domain (e.g. Heiskanen \& Moritz 1967; Wahr et al. 1998). For the representation of the gravitational potential $V$, we use the notation

$V(r, \Omega)=\frac{G M}{r} \sum_{n=0}^{\infty}\left(\frac{a}{r}\right)^{n} \sum_{m=-n}^{n} c_{n m} Y_{n m}(\Omega)$,

where $r, G$ and $M$ are the geocentric radius, the gravitational constant and the Earth mass, respectively, $Y_{n m}(\Omega)$ are the fully normalized SH base functions of degree $n$ and order $|m|$ and $c_{n m}$ are the corresponding Stokes coefficients. Positive or negative $m$ indicate cosine or sine dependence on longitude (That is, for $m \geq 0$ and $m$
$<0$, respectively, the $c_{n m}$ correspond to the $C_{l m}$ and $S_{l m}$ in Wahr et al. 1998). GRACE yields residual changes $\Delta c_{n m}^{\text {sat }}(t)$ with respect to background models of gravity field variations. The GRACE products are restricted to the satellite-derived part of the SH spectrum $\{n m\}_{\text {sat }}$ (e.g. $n=2, \ldots, 120, m=-n, \ldots, n$ for the release- 4 solutions by GFZ), and they contain errors $\varepsilon_{n m}^{\mathrm{sat}}(t)=\Delta c_{n m}^{\mathrm{sat}}(t)-$ $\Delta c_{n m}(t)$.

A mass change estimate $\widehat{\Delta m}(t)$ based on GRACE monthly solutions is expressed as a linear functional of the general form

$\widehat{\Delta m}(t)=\sum_{\{n m\}_{\mathrm{sat}}} \alpha_{n m} \Delta c_{n m}^{\mathrm{sat}}(t)$,

where the factors $\alpha_{n m}$ are to be specified.

To explore how $\widehat{\Delta m}(t)$ relates to $\Delta m(t)$, we express (1) in the $\mathrm{SH}$ domain (Wahr et al. 1998):

$\Delta m(t)=4 \pi a^{2} \sum_{\{n m\}_{\mathrm{all}}} \vartheta_{n m} \Delta \kappa_{n m}(t)$.

Here, $\sum_{\{n m\}_{\text {all }}}$ denotes the summation $\sum_{n=0}^{\infty} \sum_{m=-n}^{n}$ and $\vartheta_{n m}$, $\Delta \kappa_{n m}(t)$ are the SH coefficients of $\vartheta(\Omega), \Delta \kappa(\Omega, t)$ according to the following convention to expand a square-integrable function $f(\Omega)$ on the sphere into spherical harmonics:

$f(\Omega)=\sum_{n=0}^{\infty} \sum_{m=-n}^{n} f_{n m} Y_{n m}(\Omega)$.

By splitting and rearranging the factors, eq. (3) becomes

$\widehat{\Delta m}(t)=4 \pi a^{2} \sum_{\{n m\}_{\mathrm{sat}}} \eta_{n m} \Delta \kappa_{n m}^{\mathrm{sat}}(t)$,

where

$\Delta \kappa_{n m}^{\mathrm{sat}}(t)=K_{n} \Delta c_{n m}^{\mathrm{sat}}(t)$

are the SH coefficients of a surface density change equivalent to the satellite-derived gravity field change, which are obtained using

$K_{n}=\frac{2 n+1}{1+k_{n}^{\prime}} \frac{M}{4 \pi a^{2}}$,

with $k_{n}^{\prime}$ being the load Love numbers after Farrell (1972). In the spatial domain, (6) has the form

$\widehat{\Delta m}(t)=\iint_{\Omega} \eta(\Omega) \Delta \kappa^{\text {sat }}(\Omega, t) a^{2} \mathrm{~d} \Omega$,

where $\Delta \kappa^{\text {sat }}(\Omega, t)$ is the spatial representation of the satellite-derived surface density changes and, accordingly, $\eta(\Omega)$ is the spatial representation of the coefficients $\eta_{n m}$ following the convention (5). That is, the weight function $\eta(\Omega)$ in (9) arises from the choice of the linear factors in (3) or (6).

\subsection{Errors of mass change estimates}

Comparing (4) with (6) or (1) with (9) shows the formal structure of the error of the estimate. It is composed of two effects (Swenson \& Wahr 2002):

$\widehat{\Delta m}(t)-\Delta m(t)=\varepsilon \frac{\text { leak }}{\Delta m}(t)+\varepsilon \frac{\text { sat }}{\Delta m}(t)$.

The so-called leakage effect $\varepsilon \frac{\text { leak }}{\Delta m}$ results from the spectral truncation and the replacement of $\vartheta_{n m}$ by $\eta_{n m}$ when stepping from (4) to (6). The GRACE error effect $\varepsilon \frac{\text { sat }}{\Delta m}$ is the direct effect of errors in the GRACE gravity field solutions. Specifically,

$$
\begin{aligned}
\varepsilon \frac{\text { leak }}{\Delta m}(t)= & -4 \pi a^{2} \sum_{\{n m\}_{\text {nonsat }}} \vartheta_{n m} \Delta \kappa_{n m}(t) \\
& +4 \pi a^{2} \sum_{\{n m\}_{\text {sat }}}\left(\eta_{n m}-\vartheta_{n m}\right) \Delta \kappa_{n m}(t),
\end{aligned}
$$




$$
\begin{aligned}
\varepsilon_{\Delta m}^{\mathrm{sat}}(t) & =4 \pi a^{2} \sum_{\{n m\}_{\mathrm{sat}}} \eta_{n m}\left(\Delta \kappa_{n m}^{\mathrm{sat}}(t)-\Delta \kappa_{n m}(t)\right) \\
& =4 \pi a^{2} \sum_{\{n m\}_{\mathrm{sat}}} \eta_{n m} K_{n} \varepsilon_{n m}^{\mathrm{sat}}(t)
\end{aligned}
$$

Here, $\sum_{\{n m\}_{\text {nonsat }}}$ denotes the summation over the SH spectrum not covered by the GRACE solutions. In the spatial domain, the two error types can be expressed as

$\varepsilon_{\frac{\Delta m}{\text { leak }}}^{\text {en }}(t)=\iint_{\Omega}(\eta(\Omega)-\vartheta(\Omega)) \Delta \kappa(\Omega, t) a^{2} \mathrm{~d} \Omega$,

$\varepsilon_{\overline{\Delta m}}^{\mathrm{sat}}(t)=\iint_{\Omega} \eta(\Omega)\left(\Delta \kappa^{\mathrm{sat}}(\Omega, t)-\Delta \kappa(\Omega, t)\right) a^{2} \mathrm{~d} \Omega$.

Leakage effects are induced by any geophysical variation $\Delta \kappa(\Omega$, $t$ ), which is not part of the a priori reduced background model. In particular, in accordance with the definition by Swenson \& Wahr (2002, their eq. 35), leakage not only occurs from variations outside the region of interest (hereafter termed leakage from outside) but also from variations inside this region (leakage from inside).

The choice of a specific estimation method that determines the factors $\eta_{n m}$ or, equivalently, the weight function $\eta(\Omega)$ affects both leakage and GRACE error effects.

Commonly the mass variations $\Delta \kappa(\Omega)$ are a spatial superposition of variations in different Earth subsystems, for example, ice, atmosphere and solid earth. An interpretation of the estimated integrated mass variations in terms of one single subsystem (e.g. ice masses) must rely on a separation of the other subsystem signals, for example, by reducing respective models. Then, an additional error results from the imperfection of this separation. Note in this context that GIA, as occurring in the Earth's interior, is no surface mass process. It can be, anyway, included in the above formalism since it can be uniquely assigned to a surface mass process with identical gravity field effect.

For error assessments, errors are treated as random variables, and their quadratic mean is estimated, which is called rms error, error standard deviation (STD; if zero-centred) or just error.

\subsection{Particular estimation methods}

\subsubsection{Regional integration approach}

The regional integration approach introduced by Wahr et al. (1998) and Swenson \& Wahr (2002) starts directly from the idea of computing a regional integral (1) and takes the weight function $\eta(\Omega)$ as a filtered version of the region function $\vartheta(\Omega)$. Apart from Gaussian filtering (Wahr et al. 1998) several more sophisticated filters have been proposed.

Here we consider the Lagrange multiplier method (Swenson \& Wahr 2002), which minimizes the global mean of $(\eta(\Omega)-\vartheta(\Omega))^{2}$ under the constraint that the STD of the GRACE error effect, $\sigma\left(\varepsilon \frac{\mathrm{sat}}{\Delta m}\right)$, does not exceed a given value $\sigma_{\text {con }}$. An often employed case assumes $\sigma\left(\varepsilon \frac{\mathrm{sat}}{\Delta m}\right)$ to be modelled by uncorrelated propagation of the Stokes coefficient errors $\sigma_{\varepsilon_{n m}}$, and this results in

$\eta_{n m}=w_{n m} \vartheta_{n m} \quad$ for $(n, m) \in\{n m\}_{\text {sat }}$

with damping factors $w_{n m}$ determined by

$w_{n m}=\frac{1}{1+\lambda K_{n}^{2} \sigma_{\varepsilon_{n m}}^{2}}$, where $\lambda$ is the unique positive solution of the equation

$$
\sum_{\{n m\}_{\mathrm{sat}}} \frac{\left(4 \pi a^{2}\right)^{2} K_{n}^{2} \sigma_{\varepsilon_{n m}}^{2} \vartheta_{n m}^{2}}{\left(1+K_{n}^{2} \sigma_{\varepsilon_{n m}}^{2} \lambda\right)^{2}}=\sigma_{\mathrm{con}}^{2} \text {. }
$$

Note that the impact of $\vartheta(\Omega)$ and $\sigma_{\text {con }}$ on the filter factors $w_{n m}$ is just by the single damping parameter $\lambda$. Any value of $\lambda$ provides an optimal filter with respect to the constraint $\sigma_{\text {con }}$ related to $\lambda$ by (17). Thus, instead of starting with a value for $\sigma_{\text {con }}$ (chosen somewhat arbitrarily), one may also start with a value for $\lambda$ (chosen with the same arbitrariness) to specify the $w_{n m}$.

Although it obeys a formal optimal criterion, the Lagrange multiplier method does not appear particularly optimal, since it does not account for geographical characteristics of the mass variations of interest. For Antarctica, for example, the mass variations after reduction of modelled atmospheric and oceanic variations have a tendency to be larger on the continent than in the adjacent ocean and to be larger at the continental margin than in the continental interior. The minimization of $(\eta(\Omega)-\vartheta(\Omega))^{2}$ is globally uniform, leading to a weight function $\eta(\Omega)$ that typically deviates largely from 1 near the region boundary. In addition, for large regions such as the Antarctic continent $\eta(\Omega)$ is typically below 1 in the entire region. Methods of introducing geophysical signal characteristics have been proposed (e.g. Swenson \& Wahr 2002; Seo et al. 2006), but their current numerical realizations still use simple stochastic models of the geophysical signal, for example, not accounting for covariances between Stokes coefficients. They, thus, still do not exploit the geographical a priori information.

\subsubsection{Heuristic amendments to the regional integration approach}

The current imperfection of the regional integration approach justifies heuristic, or empirical, amendments. Here we consider two such amendments. The first one is a widening of the region function (and, hence, of the resulting weight function) of continental regions towards the ocean to increase the weight of continental mass changes near the coast. More precisely, we consider a modified region function $\zeta(\Omega)$, with $\zeta(\Omega)=1$ in the integration region, $\zeta(\Omega)=\frac{1}{2}+\frac{1}{2} \cos \left(\pi d / d_{\max }\right)$ in the adjacent ocean region and $\zeta(\Omega)=0$ everywhere else. The 'adjacent ocean region' comprises all oceanic positions with distance $d$ from the integration region being smaller than a maximum distance $d_{\max }$ (here: $400 \mathrm{~km}$ ) and smaller than the distance to any other continental region. The second amendment is the introduction of a yet unspecified scaling factor $\gamma$. In particular,

$\eta_{n m}=\gamma w_{n m} \zeta_{n m}$

\subsubsection{Fitting predefined patterns}

The formalism of Sections 2.1 and 2.2 applies not only to the regional integration approach but for any estimate having the form of a linear functional similar to (3). The general functional form may also assert certain assumptions about the signal's spatial patterns a priori. It may also include detailed geographical parameters and then retrieve the areal integral. More specifically, the mass variations are modelled as a linear combination of a finite number of given patterns. Linear factors for each of these patterns are estimated from the GRACE monthly solutions. Formally, let $\Delta \kappa_{i}(\Omega)(i=1, \ldots, u)$ be the patterns of surface load variations, and let $\left(\Delta c_{n m}\right)_{i}((n, m) \in$ $\left.\{n m\}_{\text {all }}, i=1, \ldots, u\right)$ be the related sets of Stokes coefficients in 
the sense of (7). For every $i$, the $\left(\Delta c_{n m}\right)_{i}$ with $(n, m) \in\{n, m\}_{\text {sat }}$ are arranged in a column vector $\boldsymbol{x}_{i}$. In the same way, the satellite-derived Stokes coefficient anomalies $\Delta c_{n m}^{\mathrm{sat}}(t)$ are arranged as a vector $\boldsymbol{y}(t)$ and their errors $\varepsilon_{n m}$ as a vector $\boldsymbol{\varepsilon}$ with covariance matrix $\operatorname{Cov}(\boldsymbol{\varepsilon})=$ $\sigma^{2} \boldsymbol{P}^{-1}$. This leads to the Gauss Markov model (Koch 1999)

$\boldsymbol{X} \boldsymbol{\beta}(t) \equiv \sum_{i=1}^{u} \beta_{i}(t) \boldsymbol{x}_{i}=\boldsymbol{y}(t)-\boldsymbol{\varepsilon}, \quad \operatorname{Cov}(\boldsymbol{\varepsilon})=\sigma^{2} \boldsymbol{P}^{-1}$

and the following estimate for the vector $\boldsymbol{\beta}(t)$ of the factors $\beta_{i}(t)$ :

$\hat{\boldsymbol{\beta}}(t)=\left(\boldsymbol{X}^{\prime} \boldsymbol{P} \boldsymbol{X}\right)^{-1} \boldsymbol{X}^{\prime} \boldsymbol{P} \boldsymbol{y}(t)$.

Then, estimates of integrated mass changes arise indirectly from the mass changes $\Delta m_{i}$ (arranged in the column vector $\Delta \boldsymbol{m}$ ) that are induced by the individual patterns $\Delta \kappa_{i}(\Omega)$ :

$$
\begin{aligned}
\widehat{\Delta m}(t) & =\sum_{i=1}^{u} \Delta m_{i} \hat{\beta}_{i}(t)=\boldsymbol{\Delta} \boldsymbol{m}^{\prime} \hat{\boldsymbol{\beta}}(t) \\
= & {\left[\boldsymbol{\Delta} \boldsymbol{m}^{\prime}\left(\boldsymbol{X}^{\prime} \boldsymbol{P} \boldsymbol{X}\right)^{-1} \boldsymbol{X}^{\prime} \boldsymbol{P}\right] \boldsymbol{y}(t) } \\
= & 4 \pi a^{2} \sum_{\{n m\}_{\mathrm{sat}}} \eta_{n m} K_{n} \Delta c_{n m}^{\mathrm{sat}}(t) .
\end{aligned}
$$

The equality (23) arises from expressing $\boldsymbol{y}(t)$ by its elements $\Delta c_{n m}^{\text {sat }}(t)$ and expressing the elements of the square-bracketed vector as $4 \pi a^{2} \eta_{n m} K_{n}$, thus, deducing the factors $\eta_{n m}$ of (6).

For an exact linear model (19), GRACE errors would be the only error source. However, the true mass variations are unlikely to be precisely composed of only the chosen set of spatial patterns. This causes a model error in the Gauss Markov model and, as a result, additional errors to be reconciled in the mass change estimate. In the sense of the general formalism of Section 2.2, these errors are leakage errors as described by (11) and (13).

\subsection{Previous work discussed in the methodological framework}

The regional integration approach has dominated analyses of mass changes using GRACE harmonic fields. The concept of rescaling the results ( $c f$. 'second amendment' of Section 2.3.2) has arisen from considerations of leakage from inside (e.g. Velicogna \& Wahr 2006a; Chen et al. 2007). The assessment of this leakage differs between different studies. For example, Ramillien et al. (2006) do not discuss this effect, whereas Velicogna \& Wahr (2006a) rely on the assumption of a uniform mass change over the integration region, and Chen et al. (2007) and Fenoglio-Marc et al. (2006) base their treatments on more realistic models of mass variations. Wahr et al. (2007) recently widened an earlier region function assumed by Velicogna \& Wahr (2006b), and this is similar to the 'first amendment' discussed in Section 2.3.2

The approach of fitting predefined mass change patterns (Section 2.3.3) corresponds to the general idea of using a priori information to reduce or constrain the parameters that have to be estimated to explain the satellite observations. Wiehl et al. (2005) present a conceptual study of this approach. Chen et al. (2006) and Sasgen et al. (2007) are lead by the same idea but perform the fit in the spatial domain based on filtered GRACE fields. Limiting the allowed mass variations to small areas might make it difficult to extrapolate inferences to a pan-Antarctic scale.

The approach of fitting predefined patterns has some correspondence to the mascon approach of GRACE data analysis (e.g. Luthcke et al. 2006) but our approach has to rely on the monthly global GRACE solutions as intermediate products and thus can not exploit the ability of the mascon method to employ individual passes of the satellite pair over the target region. The inverse method by Ramillien et al. $(2004,2005)$ can be viewed as a generalization of the approach of fitting predefined patterns.

\section{APPLiCATION TO ANTARCTIC MASS CHANGE}

Our mass change estimates use 61 release-4 GRACE monthly solutions by GFZ (GFZ 2007). They cover the months 08/2002 to $01 / 2008$ except 09 and $12 / 2002,01$ and $06 / 2003$ and $01 / 2004$. They are unregularized solutions of the SH degrees $n=2, \ldots, 120$, with the exception of five months (07-10/2004 and 12/2006) for which we use the regularized solutions provided by GFZ. Any statistical analyses (including the estimation of trends) will be restricted, however, to the 56 unregularized months.

The monthly solutions are provided together with monthly data sets of the atmospheric and oceanic background models. Here, when regarding these background fields, we decompose their sum into the ocean bottom pressure model that is provided as an extra product by GFZ and the rest, which is the continental atmospheric model. The modelled atmospheric and oceanic variations are already reduced from the monthly solutions. We reduce additional models of gravity field variations to isolate ice mass signals. These additional reductions are a model of Antarctic GIA, based on the ice load history of Ivins \& James (2005) and a mean mantle viscosity profile (the IJ05 model) and the Water GAP Global Hydrology Model (WGHM; Döll et al. 2003) complemented by uniform oceanic layer changes to assure global mass conservation.

We consider the following integration regions: the entire Antarctic continent (grounded ice); East Antarctica and West Antarctica (including the Antarctic Peninsula), as well as 16 major drainage basins as delineated in Fig. 1, which are roughly adopted from previous studies (Davis et al. 2005; Zwally et al. 2005; Wingham et al. 2006).

The detailed analyses through Section 4.6 are confined to two exemplary basins: entire Antarctica and Basin 12 in the Amundsen Sea sector of West Antarctica.

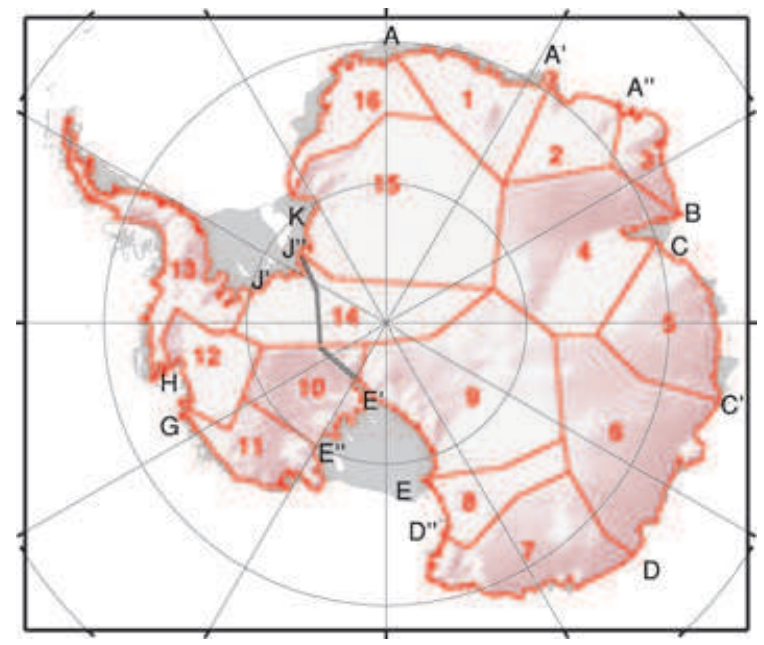

Figure 1. Antarctic integration regions considered in this study: East and West Antarctica (separated by grey line) and 16 individual drainage areas (red boundaries). 


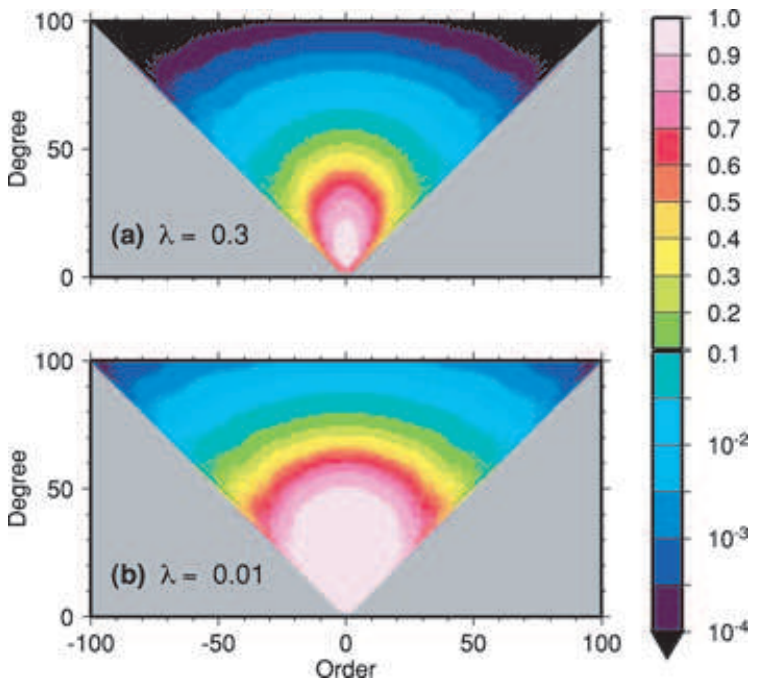

Figure 2. Damping factors $w_{n m}$ for (a) $\lambda=0.3$ and (b) $\lambda=0.01$.

The general estimation scheme (6) is implemented by three different techniques (hereafter denoted by Method I, II and III): the 'pure' Lagrange multiplier method of regional integration (Section 2.3.1), the Lagrange multiplier method with the amendments introduced in Section 2.3.2 and, finally, the method of fitting predefined spatial patterns (Section 2.3.3). When specifying $\eta_{n m}$, we use the calibrated error STDs of the Stokes coefficients provided together with the monthly solutions and take their quadratic mean values over the unregularized months.

For Methods I and II, results were generated for a range of different damping parameters $\lambda$. Likewise, different scaling parameters $\gamma$ were tested with Method II. Parameter selection is a compromise between leakage and GRACE error effects. The detailed error assessments leading to these choices are described in the next section. At this point, we just mention the final values because the illustrations and numerical examples are related: We ultimately use $\lambda=$ $0.3, \gamma=1.33$ for entire Antarctica and $\lambda=0.01, \gamma=1$ for Basin 12. As a first illustration, Fig. 2 shows the resulting damping factors $w_{n m}$ for both cases.

Method III is an initial test of the approach of fitting predefined spatial patterns. Owing to the spherical harmonic input data, the solution has to be global in nature and the involved patterns are defined globally, although we are ultimately interested in Antarctic mass changes. We use 67 patterns, each of them representing surface mass changes in a certain region complemented by a uniform ocean layer change to assure mass conservation. 16 patterns represent uniform changes over each of the 16 Antarctic drainage basins. 16 other patterns represent non-uniform changes in the 16 basins. These patterns have large values near the coast and small values in the continental interior. They account for the fact that temporal variations in both surface mass balance (SMB) and ice dynamics are typically confined to coastal regions. Specifically, the following dependence on the distance $D(\Omega)$ from the grounding line or coast:

$\Delta \kappa(\Omega)=\left(\frac{7600}{\sqrt{D(\Omega) \mathrm{km}^{-1}+50}}-199\right) \mathrm{kg} \mathrm{m}^{-2}$

is employed following Van Lipzig et al. (2002), who identified this as a reasonable approximation of the space-dependence of the SMB. The remaining 35 patterns represent uniform surface mass changes of regions outside the Antarctic continent: 32 oceanic regions (in-
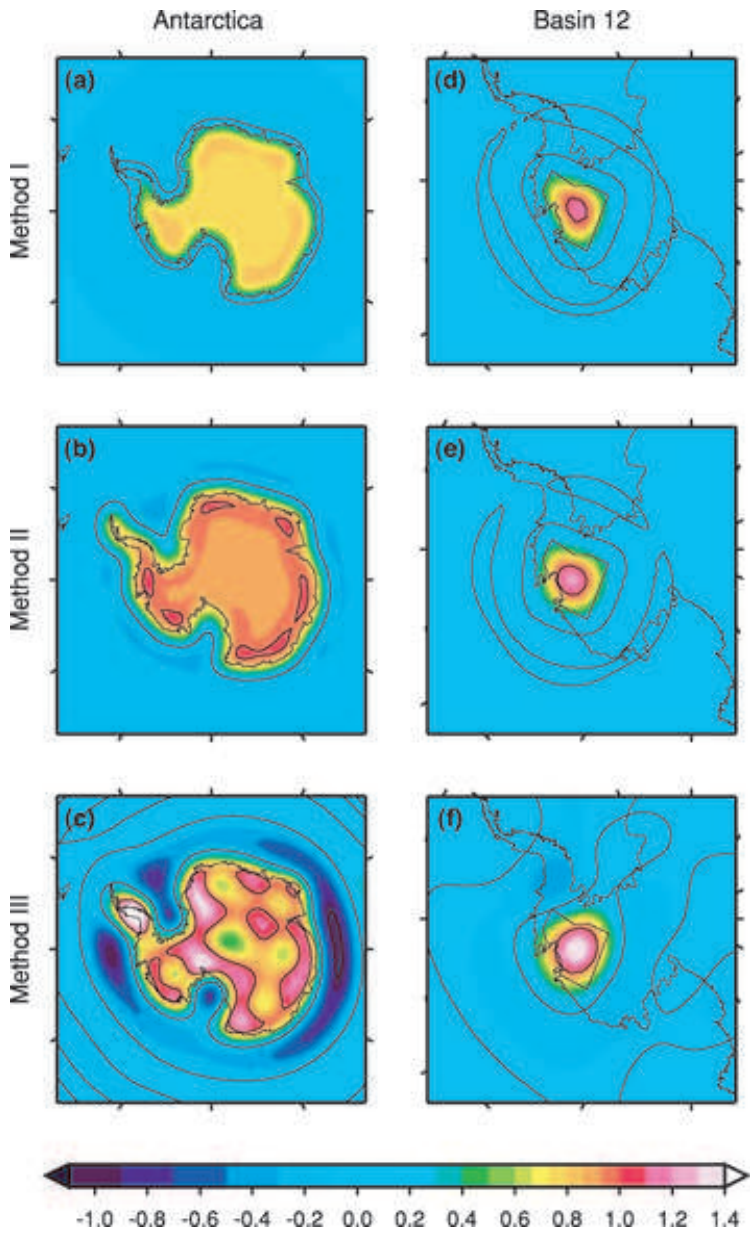

Figure 3. Weight functions $\eta(\Omega)$ used in mass change estimates for Antarctica (left-hand panel) and Basin 12 (right-hand panel) with Methods I, II and III (top, centre, bottom). Black isolines show the values $-1,0$ and 1 .

cluding ice shelves) covering the ocean approximately south of $-40^{\circ}$ latitude; the global land area excluding Antarctica and Greenland; Greenland; and a circular area with $1200 \mathrm{~km}$ radius centred in the Hudson Bay. By these patterns, variations outside Antarctica (oceanic variations, continental hydrology, Greenland ice mass variations and GIA in North America) shall be roughly parametrized and, thus, prevented from causing too large biases in the Antarctic ice mass estimates.

The arising weight functions $\eta(\Omega)$ are shown in Figs 3 and 4 to illuminate some general features of the three methods: For the case of entire Antarctica, the global patterns for Methods I and II are induced by the omission of the degree-1 components of $\vartheta(\Omega)$ and the damping of further low-degree components. For Method I, the low-degree omission and damping makes $\eta(\Omega)$ significantly smaller than 1 everywhere within the integration region. With Method II, this effect is compensated by the rescaling with $\gamma$. The damping of the high-degree components of $\vartheta(\Omega)$ yields a smooth transition of $\eta(\Omega)$ over the region boundaries. Values right at the boundary are typically around 0.3 for Method I. For Method II the widening of the original region function assures that, now, $\eta(\Omega)$ hugs a value near 1 at the coast and reduces only in the adjacent ocean. For Method III, $\eta(\Omega)$ deviates relatively strongly from $\vartheta(\Omega)$, both in the global pattern and in the Antarctic region. With this method, $\eta(\Omega)$ is retrieved more indirectly from the condition that for certain mass 

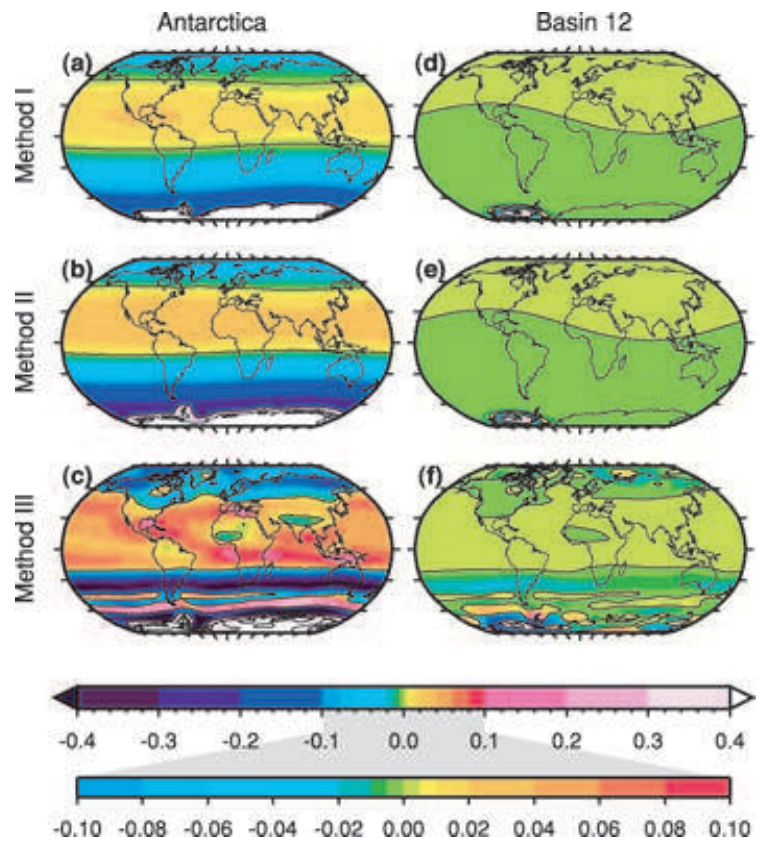

Figure 4. The same as in Fig. 3, but in a global view and with different colour scale.

variation patterns the $\eta(\Omega)$-weighted integral equals their actual integral.

For the case of Basin 12, the global patterns of $\eta(\Omega)$ have much smaller amplitudes than for all of Antarctica, since the low-degree components of $\vartheta(\Omega)$ are now much smaller. The damping of high degrees now not only leads to a smoothed transition over the region boundary but also to an overshooting in the region interior.

Time-series of estimated monthly ice mass changes are shown in Fig. 5. Linear, annual and semiannual harmonic functions (called linear and seasonal model) are fit to these time-series, where the individual months are weighted according to their GRACE error effects propagated from the calibrated errors.

\section{ERRORS OF ESTIMATED ICE MASS CHANGES}

Our error assessments focus on the error of the linear trend of the $\widehat{\Delta m}(t)$. Similar analyses may be performed for other parameters such as seasonal signal amplitudes. As a starting point, the diamonds in Fig. 6 show the formal a posteriori errors of the trend as obtained from the residuals of the linear and seasonal fit (e.g. Vaníček \& Krakiwsky 1986). The following sections describe the error synopsis given in this figure.

\subsection{Errors of superimposed mass variation corrections}

The GIA correction is a well-recognized source of error in ice mass trend estimates. Fig. 7 (brown curves) shows the effects of our GIA model reduction. For entire Antarctica, they are about as large as the finally estimated ice mass trends.

Concerning the GIA correction error for entire Antarctica we refer to Velicogna \& Wahr (2006a) who computed a range of GIA corrections based on two models of ice load history (IJ05; Ivins \& James 2005, and ICE-5G; Peltier 2004) and a realistic range of mantle viscosities. The corrections ranged from 104 to $249 \mathrm{Gt} \mathrm{yr}^{-1}$. Velicogna \& Wahr (2006a) took the midpoint of this interval as their GIA correction and the half width $\left(72.5 \mathrm{Gt} \mathrm{yr}^{-1}\right)$ as their GIA error. For the present study, we assess an error of $40 \mathrm{Gt} \mathrm{yr}^{-1}$, that is, lower by factor 1.8, for two reasons: First, the GIA correction error depends on the adopted weight function $\eta(\Omega)$ just as the correction itself does. The weight function by Velicogna \& Wahr (2006a, their fig. 1 , multiplied by $1 / 0.61$ ) is considerably larger than our weight function at positions with large GIA signals, for example, on the Ross and Filchner Ronne ice shelves and at the root of the Antarctic Peninsula. Second, recent geological evidence does not support the strong Antarctic ice losses included in ICE-5G (Ivins \& James 2005 , 2006), so that the upper range of GIA corrections obtained by Velicogna \& Wahr (2006a) appears less realistic.

For Basin 12 (Fig. 6b), the GIA correction is smaller than $4 \mathrm{Gt} \mathrm{yr}^{-1}$. We assume a $4 \mathrm{Gt} \mathrm{yr}^{-1}$ error of this correction.

A way of validating, to some extent, the IJ05 model is to look at GRACE results for the two large Antarctic ice shelves where
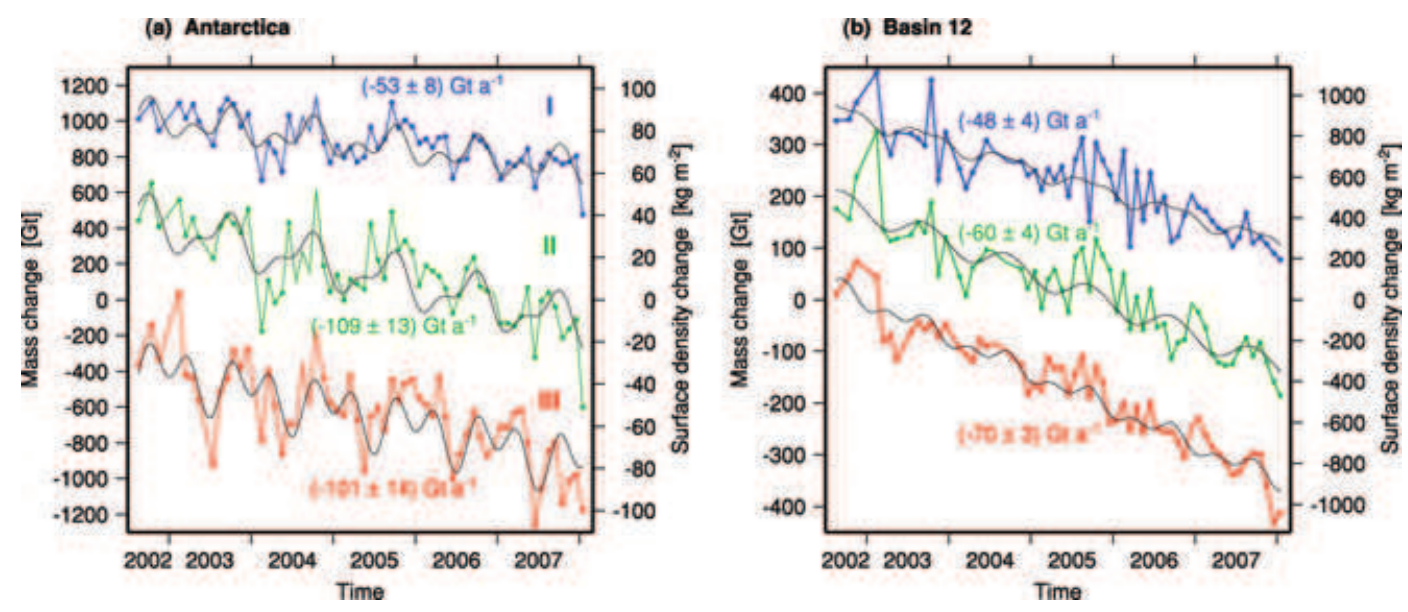

Figure 5. Estimated ice mass changes for (a) Antarctica and (b) Basin 12 with Methods I (blue), II (green) and III (red). Dots mark unregularized months. The fitted linear and seasonal models (black) and their trends with formal error STDs are also shown. The right-hand ordinate axes show the corresponding mean surface density changes over the region. Absolute levels are shifted arbitrarily. 
(a) Antarctica

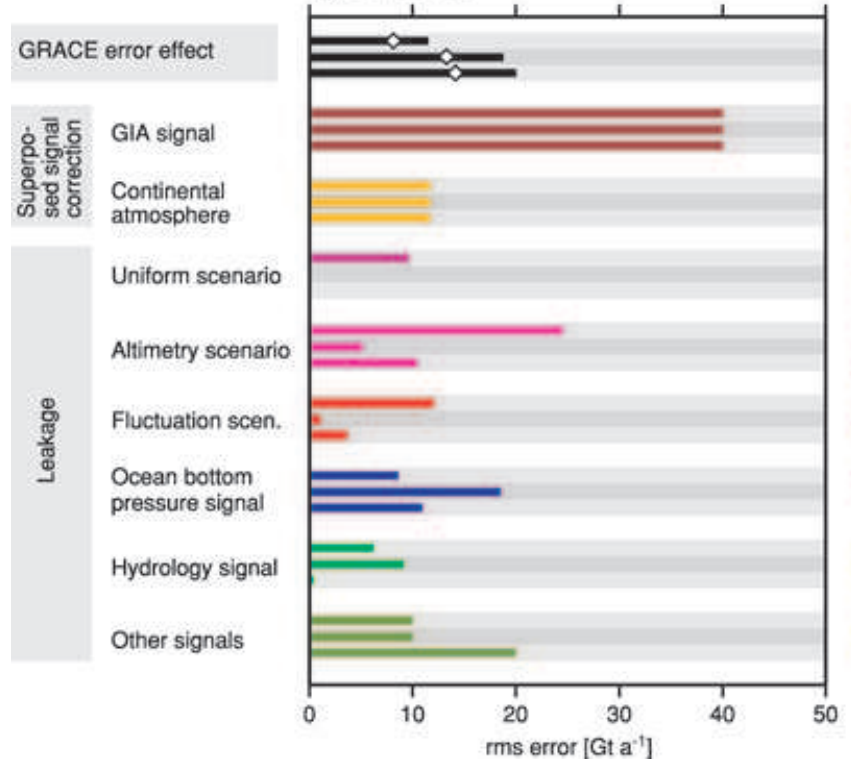

(b) Basin 12

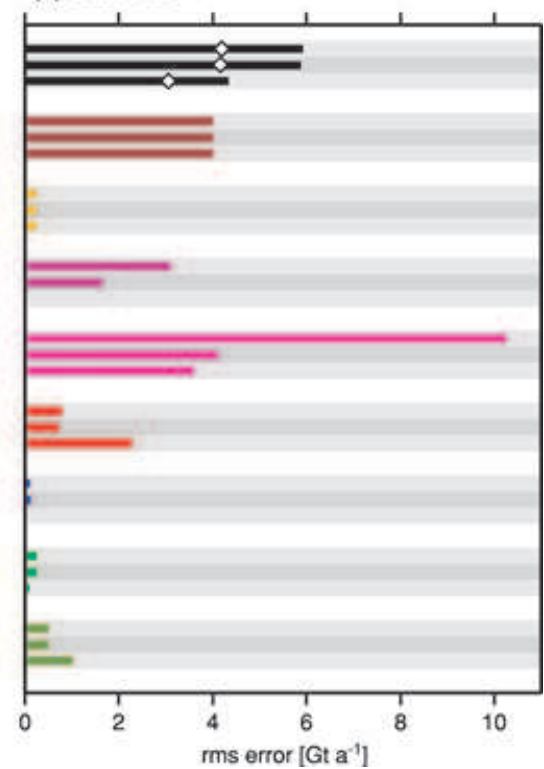

Figure 6. Error budget of estimated ice mass trends for (a) Antarctica and (b) Basin 12. Diamonds show formal a posteriori errors of the ice mass trends. Bars show assessed rms errors of different origins distinguished by colours where, each time, the three entries refer to Methods I, II and III, respectively. For the fluctuation scenarios, the average effects over the 20 scenarios are shown.

(a) Antarctica

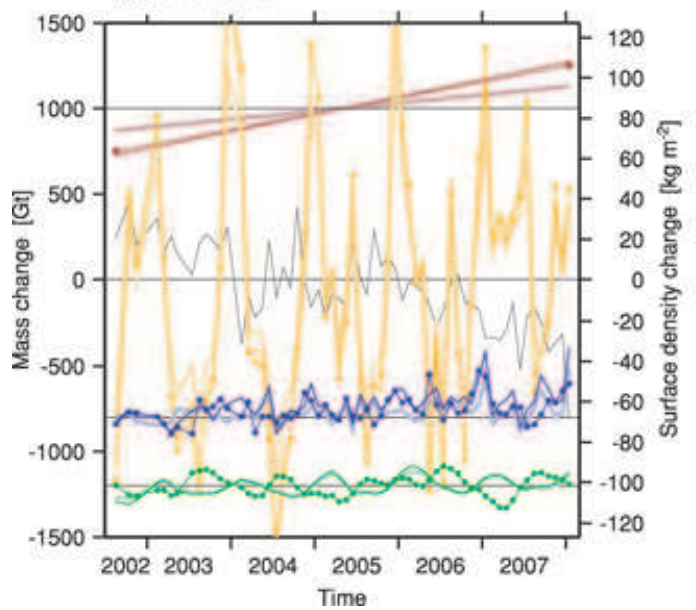

(b) Basin 12

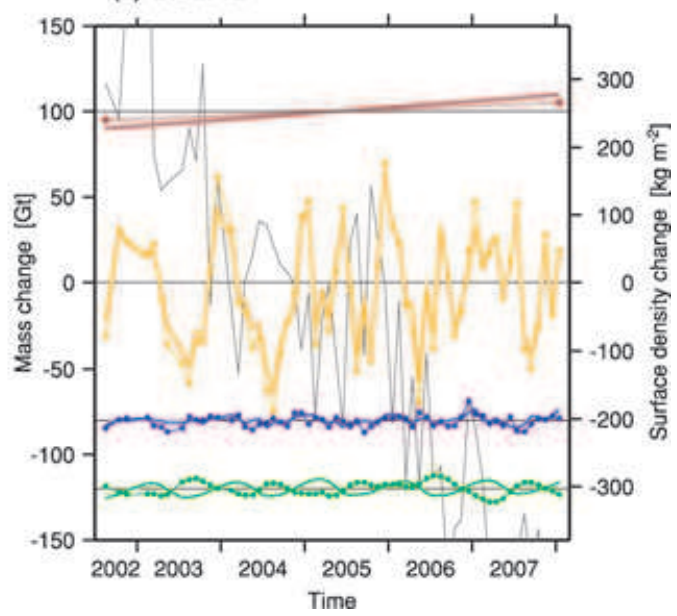

Figure 7. Reduced mass changes according to the GIA model (brown), the continental atmosphere model (orange), the ocean bottom pressure model (blue) and the hydrology model (green) as specified in Section 3. The curves show the signals that affect the ice mass change estimates had they not been reduced. For each colour, three curves (pale colour, strong colour, strong colour with dots) refer to the Methods I, II and III, respectively. For comparison, the ice mass change estimate with Method II is also shown (grey). Absolute levels are shifted arbitrarily.

modelled GIA is relatively large. With Method I applied to the regions of these ice shelves, the GIA effect after IJ05 is 7.2 and $10.3 \mathrm{Gt} \mathrm{yr}^{-1}$ for the Ross and the Filchner Ronne Ice Shelf, respectively. The residual mass change estimates from GRACE (i.e. after correction of our background models including the GIA model) should ideally be zero, as the hydrostasy of ice mass change in the ice shelf has no gravitational effect. In fact, the residual trends are as low as $(0.0 \pm 1.3)$ and $(1.4 \pm 1.4) \mathrm{Gt} \mathrm{yr}^{-1}$, respectively. These results support the IJ05 GIA model.

Atmospheric mass variations above the continent are another superimposed signal that is corrected from the estimate but subject to errors. Fig. 7 (orange curves) illustrates the effect of the continental atmospheric model reduction (see Section 3). For Antarctica, the atmospheric time-series show the largest variations among the dif- ferent signals in Fig. 7(a). The fact that the ice mass time-series vary much less (about 25 per cent STD) indicates relative good accuracy (say, at least 20 per cent) of the atmospheric reduction. We take 20 per cent as our estimate for the atmospheric correction error. This is probably too pessimistic with respect to month-to-month variations. Concerning trends, however, it is preferable to be conservative since systematic effects, for example, by changes in the underlying operational atmospheric analysis, cannot be completely ruled out.

\subsection{Leakage effects}

Assessments of leakage effects are based on simulations with model scenarios that have geographical specificity. Antarctic ice mass 
variations as an inner-Antarctic source of leakage are treated next (Section 4.2.1). Section 4.2.2 then addresses leakage from mass variations outside the Antarctic continent.

\subsubsection{Leakage induced by Antarctic ice mass variations}

First, leakage may be determined for the simplistic scenario of a uniform surface density change over the entire grounded ice sheet (or 'uniform scenario'). To retain global mass conservation, we add a compensating mass change to the global ocean. With Method I for entire Antarctica, the leakage effect induced by this scenario is -28.1 per cent of the assumed 'true' mass signal. The effect is, in turn, composed of -29.6 per cent induced by the Antarctic ice layer itself (leakage from inside) and +1.5 per cent induced by the complementing oceanic mass layer.

Roughly speaking, the integral signal is damped because $\eta(\Omega)<1$ in the entire integration region. It might be intuitive to always expect such a damping effect if $0<\eta(\Omega)<1$ in the entire integration region (e.g. Velicogna \& Wahr 2006a). However, this intuition is misleading for the general case in which mass variations occur with opposite sign. For a simple illustration, assume mass variations concentrated at two locations $\Omega_{1}, \Omega_{2}$, where the weight function $\eta(\Omega)$ happens to take the values $\eta\left(\Omega_{1}\right)=1$ and $\eta\left(\Omega_{2}\right)=$ 0.5 , respectively. If now, for example, the mass changes are -30 and $+20 \mathrm{Gt}$ at $\Omega_{1}$ and $\Omega_{2}$, respectively, then the true total mass change is $-10 \mathrm{Gt}$. However, the estimated mass change due to the different weighting is $-20 \mathrm{Gt}$. That is, the total change is amplified, not damped. If the mass changes at $\Omega_{1}$ and $\Omega_{2}$ are +30 and $-40 \mathrm{Gt}$, the true total mass change is again $-10 \mathrm{Gt}$, whereas the estimated mass change is $+10 \mathrm{Gt}$, in fact of opposite sign.

A leakage error assessment should, hence, be based on a wider and more realistic range of scenarios than just the uniform scenario. To provide such scenarios for our simulations, we resort to auxiliary data sets with a more realistic spatial correlation behaviour. These data sets do not represent 'true' ice mass trends and are not intended to be compared with GRACE results. They are just a basis for simulations. The 'altimetry scenario' (Fig. 8a) is derived from surface height trends obtained from radar altimetry of the European Remote Sensing (ERS) satellites (Legrésy et al. 2006). The 'fluctuation scenarios' (20 in total, three shown in Fig. 8b) ought to mimic the effect of interannual surface mass balance (SMB) fluctuations on a $5 \mathrm{yr}$ trend. They are randomly generated from a stochastic model, which is described in Appendix.

Fig. 9 shows the simulation results for the uniform, altimetry and fluctuation scenarios. For entire Antarctica, Method I typically suffers negative relative leakage effects, i.e., signal damping. The same is true for Method II for choice of no rescaling $(\gamma=1)$.
For Method II $(\gamma=1)$, this damping is much more consistent over all scenarios than for Method I. With Method III, there is no tendency of signal damping. For Basin 12, the tendency of damping with Methods I and II $(\gamma=1)$ is less pronounced than for entire Antarctica because now leakage from inside is superimposed by leakage from other Antarctic basins.

We now consider Method II with a rescaling by $\gamma>1$ because, at least for entire Antarctica, the dampening nature of the leakage effect is relatively consistent over all scenarios. Here, $\gamma$ is chosen to exactly compensate the leakage effect for the uniform scenario, which yields $\gamma=1.33$ for entire Antarctica and $\gamma=1.04$ for Basin 12. For entire Antarctica, this rescaling largely mitigates leakage for all scenarios. For Basin 12, leakage is mitigated in the altimetry scenario but aggravated, on average, in the fluctuation scenarios. Consequently, the standard realization of Method II employs $\gamma=1.33$ for entire Antarctica but employs no rescaling for Basin 12 .

\subsubsection{Leakage from mass variations outside Antarctica}

Modelled effects of oceanic and hydrological mass variations were reduced from the ice mass change estimates (see Section 3). These reductions are illustrated in Fig. 7. It should be mentioned that the oceanic mass compensation of hydrology constitutes a significant part of the so-called hydrological reduction.

A task remains to assess the leakage effects of errors in the reduction models. The ratio between true geophysical effects and errors in the reductions are poorly known. Hence, we make the simple working assumption that 50 per cent of the variance of our reductions are due to true geophysical effects whereas the other 50 per cent are due to model errors. This is supported by a comparison of our hydrological corrections based on the WGHM model with analogous corrections based on two alternative models by Rodell et al. (2004) and Milly \& Shmakin (2002): The corrections based on these three models exhibit different trends, and the mutual differences are typically in the order of the WGHM-derived trend. Based on the above 50 per cent assumption, the effects of oceanographic and hydrological model errors (blue and green bars in Fig. 6) are assessed as $1 / \sqrt{2}$ times the respective trends.

Further sources of leakage exist: A Greenland ice mass trend of $-200 \mathrm{Gt} \mathrm{yr}^{-1}$ (e.g. Solomon et al. 2007) induces leakage in the order of $1 \mathrm{Gt} \mathrm{yr}^{-1}$ or less. To assess leakage effects from northern hemisphere GIA, we convert geoid trends after the ICE-4G global GIA model (Peltier 1998) to equivalent surface mass trends and restrict them to the northern hemisphere. The resulting leakage effects for entire Antarctica are $-5,-7$ and $-22 \mathrm{Gt} \mathrm{yr}^{-1}$ with Methods, I, II and III, respectively. Although these results are too coarse for a correction, they indicate the magnitude of leakage from uncorrected
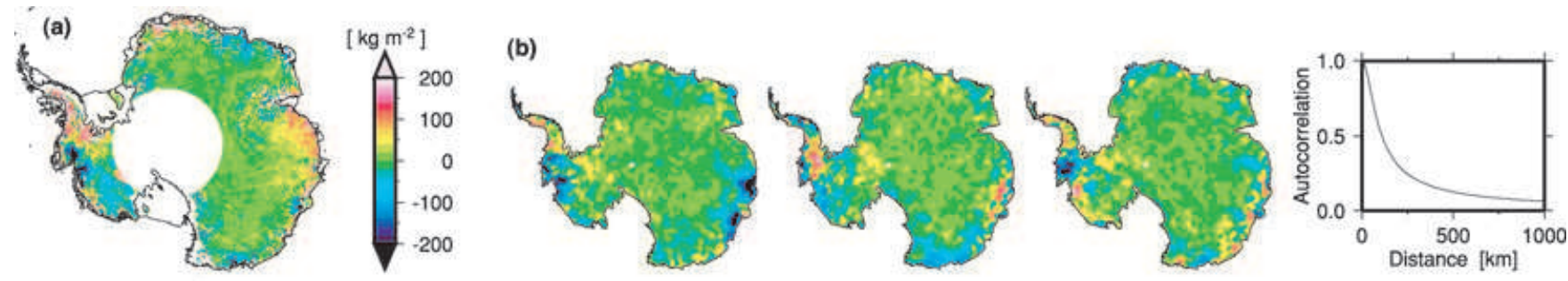

Figure 8. Auxiliary data sets of Antarctic ice mass changes used for leakage error simulations. (a) Yearly changes according to ERS-1/ERS-2 radar altimetry trends for 1995-2003 (Legrésy et al. 2006), using the simplifying assumption that the observed volume changes reflect pure ice volume changes. (b) Illustration of the stochastic model of interannual surface mass balance fluctuations: three pseudo-random realizations of yearly absolute fluctuations and the spatial autocorrelation function of relative fluctuations. 
(a) Antarctica

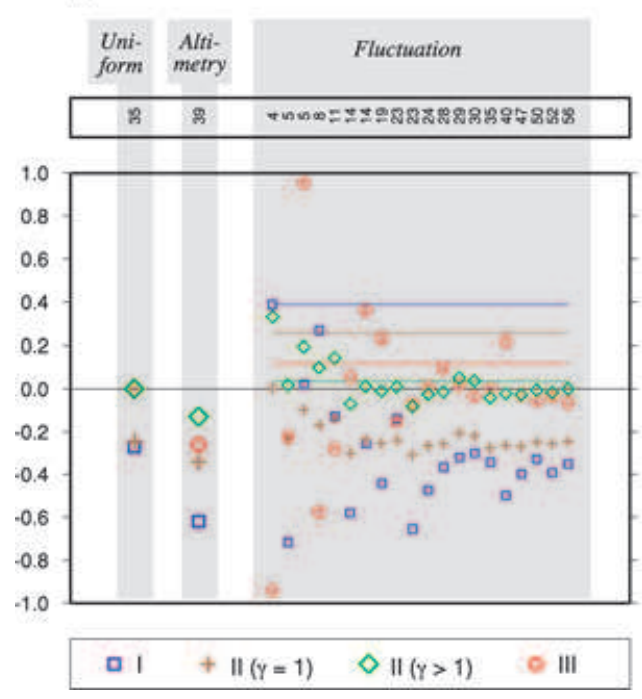

(b) Basin 12
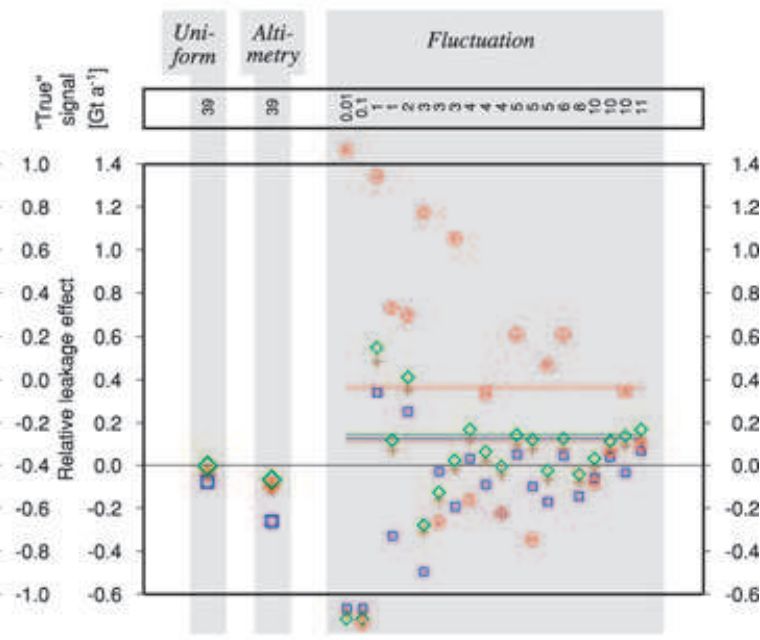

Figure 9. Simulated leakage effects of the uniform, altimetry and fluctuation scenarios for (a) Antarctica and (b) Basin 12 and for Method I (blue), Method II with $\gamma=1$ (brown), Method II with $\gamma>1$ (green) and Method III (red). Absolute values of the assumed 'true' change are quoted in the top. Leakage effects are shown relative to these 'true' changes. Coloured lines mark the average effect over the 20 fluctuation scenarios (defined as the rms absolute leakage divided by the rms 'true change'). Symbols outside the frame indicate values outside the ordinate range.

signals outside Antarctica. The respective orders of magnitude are listed as 'other signals' in Fig. 6.

\subsection{GRACE error effects}

\subsubsection{Empirical GRACE errors}

Before investigating how errors in the GRACE monthly solutions affect mass change estimates, we extend the empirical assessments of these monthly solution errors performed previously (Horwath \& Dietrich 2006). Linear and seasonal models (see Section 3) are fit to the sequence of monthly solutions, and we analyse the residuals of this fit to assess GRACE errors. The resultant STDs, over all months (corrected for noise reduction by the model fit) are termed 'empirical error' (EE) STDs and because they still contain geophysical effects, they may be considered as upper error bounds.

Fig. 10(a) shows that the EE and the calibrated errors published by GFZ have differences that exhibit an order band structure. Even more important than the coefficient-wise differences are the intercoefficient correlations detectable through the empirical analysis (Swenson \& Wahr 2006; Horwath \& Dietrich 2006). Strong correlations appear among the coefficients of the same order and of degrees with equal parity. This is evident from the empirical correlation matrix as shown in Fig. 11.

The GRACE processing centres like GFZ distribute no information on error correlations. Therefore, the standard approach of modelling GRACE error effects in spatial functionals is an uncorrelated propagation of the calibrated errors (the uncorrelated error model, or UEM). To show the actual effect of the error correlations, the EE formalism is again applied to determine the residuals of linear and seasonal fits, now performed pointwise in the spatial domain. The considered spatial functionals are the GRACE-derived surface mass density variations $\Delta \kappa^{\text {sat }}(\Omega, t)$ filtered with the $\mathrm{SH}$ factors $w_{n m}$, as applied with Methods I and II for entire Antarctica. Fig. 12(a) compares the obtained EE STDs with the error STDs according to the UEM. Some regional features in the EE, for example, in the Amazon region, can be attributed to residual geophysical
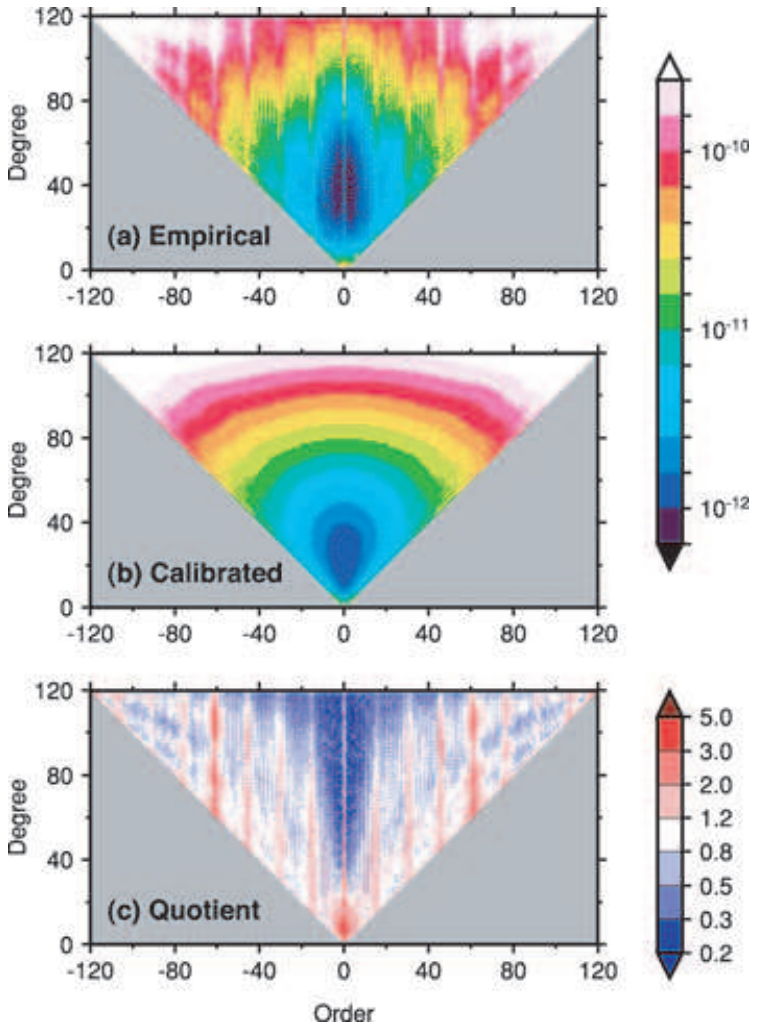

Figure 10. (a) Empirical versus (b) calibrated error STDs computed from the 56 unregularized monthly solutions. (c) Quotient between empirical and calibrated error STDs.

signals. Apart from these signals and the meridional striping, the EE differ from the UEM in their large-scale latitudinal dependence. In particular, at $60^{\circ}-85^{\circ}$ northern or southern latitude, EE are considerably larger than UEM errors, with quotients around 1.7. As argued by Horwath \& Dietrich (2006), the differences in the latitude 


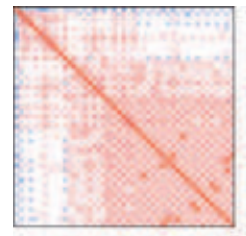

$m=6$

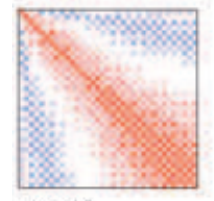

$m=16$

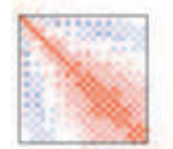

$m=26$

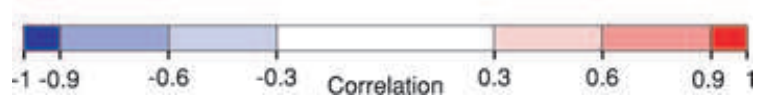

Figure 11. Sections of the empirical error correlation matrix computed from the 56 unregularized monthly solutions. Each subplot shows correlations for a fixed order $m$ and for degrees $n=m, \ldots, 50$.

distribution of errors are due to the error correlations not represented by the UEM.

\subsubsection{GRACE error effects on Antarctic mass changes}

We now turn to the effect of GRACE errors on integrated mass changes and, most importantly, on their trends. Table 1 summarizes our assessment. By pure error propagation of the UEM, the a priori error of the Antarctic trend is $5.7 \mathrm{Gt} \mathrm{yr}^{-1}$ (line $\mathrm{b}$ of the table). The a posteriori error, that is, obtained by evaluating the residuals of the linear and seasonal fit to the monthly mass changes, is as large as $13.3 \mathrm{Gt} \mathrm{yr}^{-1}$ (line c). Note that this value is affected not only by GRACE error effects but also by non-seasonal geophysical variations. Corresponding assessments are given in lines $\mathrm{d}$, e and f. For oceanic and hydrological effects the working assumption of Section 4.2.2, that is, that half of the variance of the reduction is due to model errors, is employed. Assessment of the effect of nonlinear, non-seasonal ice mass variations is based on the stochastic model of SMB fluctuations (see Appendix). After reducing the geophysical contributions (lines d, e, f) from the a posteriori error (reduction in terms of variance subtraction), there remains a residual error of $10.9 \mathrm{Gt} \mathrm{yr}^{-1}$, which now has to be attributed mainly to GRACE errors. Although it may contain atmospheric reduction errors, we argue below that these effects are secondary. Hence, the actual GRACE error effects are larger than their a priori estimates based on the UEM by about the factor 1.9 (line h).

This discrepancy is explained by the error correlations evidenced in Section 4.3.1 Indeed, for Method II, eq. (6) reads

$\widehat{\Delta m}(t)=4 \pi a^{2} \gamma \sum_{\{n m\}_{\mathrm{sat}}} \zeta_{n m} w_{n m} \Delta \kappa_{n m}^{\mathrm{sat}}(t)$

and we may interpret the factors $w_{n m}$ as a filtering of $\Delta \kappa^{\text {sat }}$. Hence, GRACE error effects in $\widehat{\Delta m}(t)$ correspond to the errors as considered in Fig. 12, integrated with the widened (but unfiltered) region function $\zeta$. These errors were found to be significantly larger than predicted by the UEM. (a) Empirical

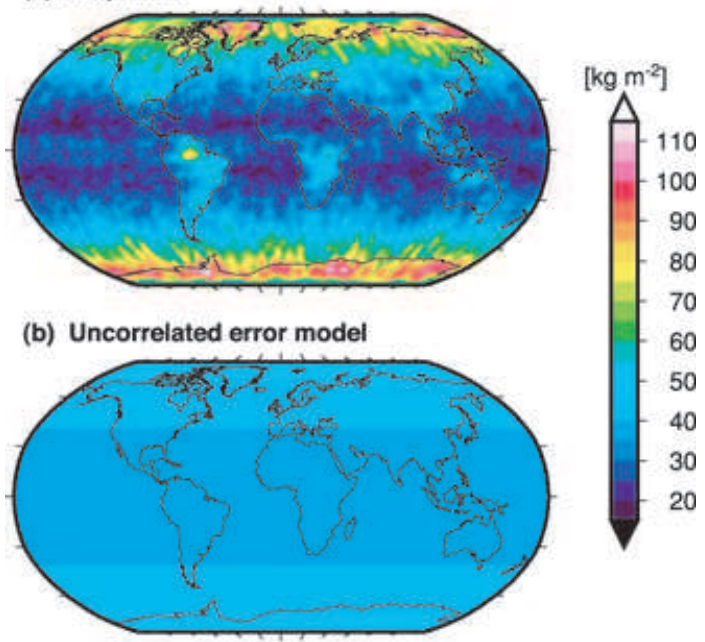

(c) Quotient

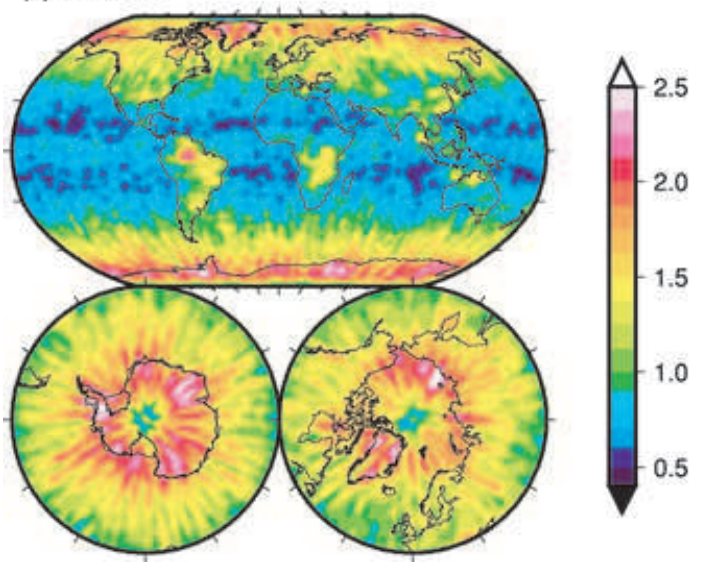

Figure 12. (a) Empirical error STDs of surface density variations filtered with the factors $w_{n m}$ as used by Methods I and II for Antarctica. (b) Error STDs for the same surface density variations according to the uncorrelated error model. (c) Quotient between empirical and uncorrelatedly modelled errors (global map and polar regions).

Table 1. Assessment of errors in the estimated ice mass trends for Antarctica and Basin 12 with Method II.

\begin{tabular}{lrrr}
\hline & & Antarctica & Basin 12 \\
\hline (a) A priori error of single month & $(\mathrm{Gt})$ & 68.9 & 20.0 \\
(b) A priori error of trend & $\left(\mathrm{Gt} \mathrm{yr}^{-1}\right)$ & 5.7 & 1.7 \\
(c) A posteriori error of trend & $\left(\mathrm{Gt} \mathrm{yr}^{-1}\right)$ & 13.3 & 4.2 \\
$\begin{array}{l}\text { (d) Contribution by ocean } \\
\text { (e) Contribution by hydrology }\end{array}$ & $\left(\mathrm{Gt} \mathrm{yr}^{-1}\right)$ & 4.6 & 0.1 \\
$\begin{array}{l}\text { (f) Contribution by ice mass } \\
\text { fluctuations }\end{array}$ & $\left(\mathrm{Gt} \mathrm{yr}^{-1}\right)$ & 1.3 & 0.0 \\
(g) Residual error, attributed to & $\left(\mathrm{Gt} \mathrm{yr}^{-1}\right)$ & 6.0 & 1.2 \\
$\quad$ GRACE and atmosphere & & 10.9 & 4.0 \\
(h) Quotient (d)/(b) & & & \\
\hline
\end{tabular}

Our assertion that residual errors associated with poorly modelled atmospheric variability are relatively benign is supported by the spatial structure of the EE in Fig. 12. Both the large-scale latitudinal dependence and the smaller-scale meridional striping seem unaffected by Arctic and Antarctic coastlines. Atmospheric effects, instead, would mainly act over land and would be inversebarometrically compensated over the ocean. 

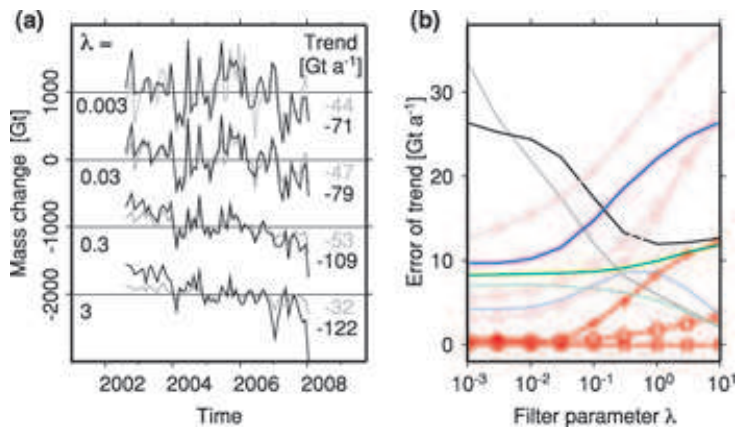

Figure 13. Influence of the filter parameter $\lambda$ for Method I (grey and faint colours) and Method II (black and bold colours) for entire Antarctica. (a) Time-series (with arbitrary absolute levels) and trends of estimated ice mass changes with different $\lambda$. (b) Influence of $\lambda$ on estimated errors: a posteriori error (grey/black), leakage effect by the uniform; the altimetry and the fluctuation scenarios (red squares, crosses and circles, respectively), leakage effects by errors of the oceanic (blue) and hydrological (green) reduction.

This assessment implies that the deviations of the monthly mass change estimates from the linear and seasonal model are dominated by GRACE errors. This fact motivates weighting the individual months according to their GRACE error level when fitting this model (see end of Section 3).

\subsection{Methodological choices}

For Methods I and II, the choice of the ultimate filter parameter $\lambda$ was made based on the error assessments described in the previous subsections performed for a range of $\lambda$ values.

Fig. 13 illustrates this choice for the case of entire Antarctica. Fig. 13(a) shows how the damping of errors and signals gets stronger with increasing $\lambda$, for Method I. For Method II, this damping is partly compensated by the rescaling with $\gamma>1$, which is always chosen such that it compensates leakage effects for the uniform scenario (see Section 4.2.1). Fig. 13(b) shows the $\lambda$-dependence of errors in the trend. With Method I, the impact of $\lambda$ is largest for the GRACE error effects and the leakage effects by Antarctic ice mass variations. A choice around $\lambda=0.3$ (the rough intersect between the bundle of faint red curves and the grey curve) seems an appropriate compromise between both types of errors. For Method II, leakage from Antarctic ice mass variations is considerably smaller whereas leakage from oceanic and hydrological reduction errors grows in importance. The choice of $\lambda=0.3$ again appears appropriate. In any case, the choice is somewhat arbitrary, governed by the models used in the error assessment.

Fig. 14 is analogous to Fig. 13 but refers to Basin $12(\gamma=1$ is fixed for Method II). The errors are now dominated by GRACE error and
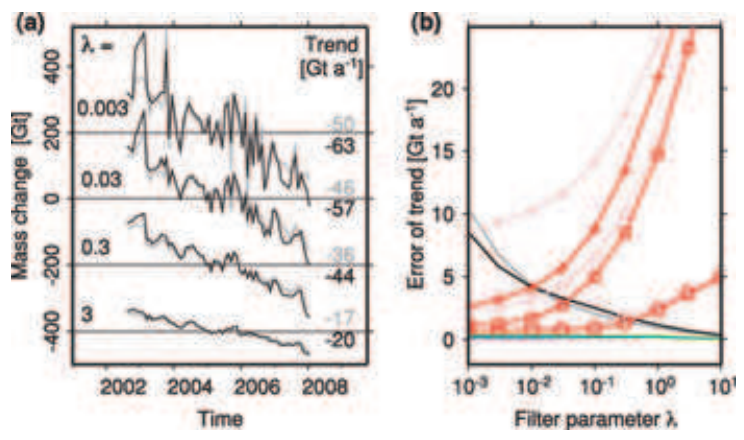

Figure 14. Same as Fig. 13 but for Basin 12. leakage effects from Antarctic ice mass variations. $\lambda=0.01$ seems a reasonable compromise between both. This choice is critically influenced by the a priori information on large ice mass changes in the region incorporated into the leakage error assessment.

For Method III, the precise definition of the Gauss Markov model strongly influences the results. For example, we tested a model modification that omits the 16 patterns representing geographically dependent mass variations within the continent. As a result, the pan-Antarctic ice mass trend reduced to -55 from $-101 \mathrm{Gt} \mathrm{yr}^{-1}$. The geographically-dependent patterns are consistent with independent observation supporting that ice mass variability is primarily coastal. The geographical dependence suggests that the weight function $\eta(\Omega)$ be close to 1 at the coast. By their omission, in contrast, only about 50 per cent of actual ice mass variations are correctly attributed to ice masses, whereas another 50 per cent are attributed to the ocean. This simple example shows the sensitivity of Method III to the predefined patterns.

\subsection{Interannual variations: signal and error}

The presence of interannual components in the mass change timeseries limits, to some extent, the ultimate value of linear trends determined in such a short time-series. By geophysical interannual variations, the trend over a few years differs from a long-term trend. For a simple assessment, assume that yearly SMB fluctuations over the entire grounded ice sheet have a relative STD of 6 per cent (cf. Van Lipzig et al. 2002; Monaghan et al. 2006). Then, with a roughly 2000 Gt mean yearly SMB (Bentley 2004), the STD of fluctuationinduced mass changes over five years is $\sqrt{5} \times 6$ per cent $\times 2000$ $\mathrm{Gt}=268 \mathrm{Gt}$, or $54 \mathrm{Gt} \mathrm{yr}^{-1}$.

A subtlety of the analysis presented in this paper is that an additional complication arises, for in fact, GRACE errors correlated in time may be another source of interannual variation and undermine the ability to determine trends. Although our GRACE error assessment so far is based on the standard assumption of temporally uncorrelated errors, this assumption is questionable. Tidal aliasing is a known source of temporally correlated GRACE errors (Seo et al. 2008; Moore \& King 2008). As another example, Horwath et al. (2007b) and Horwath (2007) report statistically significant trends in the higher degrees of GRACE monthly solutions, which resemble neither geophysical effects nor expected tidal aliasing patterns. To avoid our error assessment from being too optimistic, we include a tentative assessment of temporal correlation effects: Analyses of basin-wise mass change time-series (Horwath 2007) show that the interannual signal not explained by modelled geophysical effects is typically twice as large (in terms of variance) as random interannual effects of temporally uncorrelated errors. Hence, we estimate that the additional error variance of the trend, induced by temporal GRACE error correlations, is equal to the a posteriori error variance of the trend.

\subsection{Synopsis}

Analogously as for Antarctica and Basin 12, errors were assessed for Basins 1-16, for East and West Antarctica. Among the three considered estimation methods, Method II proved most valuable, mainly due to its relatively low leakage effects from Antarctic ice mass variations.

For the entire Antarctic continent (with $\lambda=0.3$ and $\gamma=1.33$, chosen as explained in Sections 4.4 and 4.2.1) an ice mass trend error budget was assessed as in Fig. 6. The effect of temporally

(C) 2009 The Authors, GJI, 177, 849-864

Journal compilation (c) 2009 RAS 
uncorrelated GRACE errors (as represented by the a posteriori error of the trend) is $13.3 \mathrm{Gt} \mathrm{yr}^{-1}$ (Section 4.3.2). About the same error can be assigned to leakage effects, which are dominated by oceanic and hydrological variations and possibly other variations distant from Antarctica (Section 4.2.2). Once more the same error is attributed to possible temporal correlations in GRACE errors (Section 4.5). Together, the listed error effects have an rms of $\sqrt{3} \times 13.3 \mathrm{Gt} \mathrm{yr}^{-1}$ $=23.0 \mathrm{Gt} \mathrm{yr}^{-1}$. The error in the superimposed signal correction is $40 \mathrm{Gt} \mathrm{yr}^{-1}$ for GIA and, in a conservative assessment, another $11 \mathrm{Gt} \mathrm{yr}^{-1}$ for the atmosphere (Section 4.1). The total error hence becomes $48 \mathrm{Gt} \mathrm{yr}^{-1}$. Although the GIA error dominates, other types of error, taken together, are non-negligible. In fact, the relative suppression of the other errors arises only with the extended GRACE time-series. In a previous assessment (Horwath 2007) with about 4 yr of GRACE data the GIA error and the combined effect of other errors were similar.

The error assessments for the other integration regions are performed in a similar manner and lead to the choice of $\lambda=0.01, \gamma=$ 1 for Basins 11-13, $\lambda=0.3, \gamma=1.30$ for East Antarctica and $\lambda=$ $0.3, \gamma=1$ for the remaining basins. In particular, a choice of $\gamma>$ 1 for West Antarctica did not seem justified based on leakage error simulations as in Section 4.2.1 The GIA error is difficult to assess without region-specific modelling studies. The GIA reductions tend to be proportional to the region size with about a rate of $10 \mathrm{Gt} \mathrm{yr}^{-1}$ per $10^{6} \mathrm{~km}^{2}$, and we generally assume that the GIA error follows this proportionality. This assessment may be too pessimistic and may be replaced by improved assessments where possible. Therefore, we will quote error measures with and without the GIA error.

A comparison of results from different methods shows the uncertainties that are still related to choices of methodology. Different methods imply different GRACE error and leakage effects. For example, the oceanic and hydrological reductions are very different for Methods II and III (Fig. 7). For Basin 12, the difference between Methods II and III is $9 \mathrm{Gt} \mathrm{yr}^{-1}$ whereas the assessed error (with exclusion of the GIA error) is about $7 \mathrm{Gt} \mathrm{yr}^{-1}$ for both methods.

In fact, for Basins 11-13, where ice mass changes are particularly high, leakage due to these ice mass changes, or in other words, the separation of the neighbouring basins, is crucial. As a consequence, we applied a very moderate smoothing $(\lambda=0.01)$ for these basins. Leakage is probably less crucial if we consider the Basins 11,12 and 13 together as an integration region wherein a somewhat stronger smoothing with $\lambda=0.03$ applies.

Methodological uncertainties also manifest themselves in the fact that, for Method II, the sum of estimates for individual basins is not equal to the estimate for the union of these basins. This is particularly striking for East, West and entire Antarctica (numbers will be quoted in the next section) and also occurs in previous studies such as by Velicogna \& Wahr (2006a), although less severely. The reason lies in the different filtering (implied by different $\lambda$ and $\gamma$ ) applied to the different regions according to individual, region-specific assessments. Such inconsistencies do not occur with Method III, which determines mass changes of all regions in a single inversion.

\section{ANTARCTIC ICE MASS CHANGES: RESULTS AND DISCUSSION}

Fig. 15 shows the estimated monthly ice mass variations and the ice mass trends for entire Antarctica (grounded ice) and for the 16 individual drainage basins. The Antarctic trend over the period 08/2002

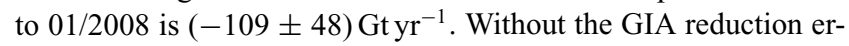

ror, the rms error would be $26 \mathrm{Gt} \mathrm{yr}^{-1}$ instead of $48 \mathrm{Gt} \mathrm{yr}^{-1}$. The trend corresponds to a mass deficit of 5 per cent of the SMB of the grounded ice sheet and it contributes $(+0.30 \pm 0.13) \mathrm{mm} \mathrm{yr}^{-1}$ eustatic sea level rise. The results for the 16 drainage basins show that the largest ice loss comes from Basin 12 (the region of the Pine Island and Thwaites Glaciers in the Amundsen Sea Embayment) with $(-60 \pm 8) \mathrm{Gt} \mathrm{yr}^{-1}$, followed by Basin 11 (northwest Marie Byrd Land) with $(-28 \pm 6) \mathrm{Gt} \mathrm{yr}^{-1}$. Basin 13 (Antarctic Peninsula and part of Ellsworth Land) contributes another large yet, according to our analysis, statistically insignificant loss of $(-19 \pm 28) \mathrm{Gt} \mathrm{yr}^{-1}$. Since the separation between these three basins is critical, as discussed in Section 4.6, we add results for their union. There, the

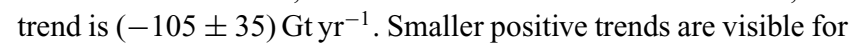
Basins 10 and 3 . The remaining basins show no significant ice mass trends over 08/2002 to 01/2008. Our estimates for East and West Antarctica are $(-1 \pm 40)$ and $(-82 \pm 42) \mathrm{Gt} \mathrm{yr}^{-1}$, respectively. The apparent discrepancy between the sum of these two trends and the pan-Antarctic trend is due to the methodological uncertainties inherent to the regional integration approach, as discussed in Section 4.6.

The strong negative trend in Basin 12 is in agreement with evidence for dynamic ice mass losses from independent observational techniques: Rignot et al. (2008) obtained (-64 \pm 27$) \mathrm{Gt} \mathrm{yr}^{-1}$ in 2000 and $(-90 \pm 27) \mathrm{Gt} \mathrm{yr}^{-1}$ in 2006 for this basin, from a mass flux budgeting method. Analyses of ERS-1/ERS-2 radar altimeter data (mostly preceding the GRACE period) inferred negative mass trends of tens of gigatons per year (up to $-63 \mathrm{Gt} \mathrm{yr}^{-1}$ ), dependent on the particular study and on density assumptions (Zwally et al. 2005; Davis et al. 2005; Wingham et al. 2006; Helsen et al. 2008).

For Basin 11, Rignot et al. (2008) report $(-23 \pm 39) \mathrm{Gt} \mathrm{yr}^{-1}$, which is, again, in agreement with our result.

The large variability of our time-series for Basin 13 is partly due to aliasing of residual ocean tides (Han et al. 2005; Moore \& King 2008), for example, with a $161 \mathrm{~d}$ alias period of the S2 tide, and fostered by the weakness of our smoothing. The negative 'trend' appears to be due to an abrupt change in the beginning of 2007. By closer analyses (not shown) we may attribute this change mainly to the tip of the Antarctic Peninsula, where Rignot et al. (2008) report negative changes of $(-15 \pm 8) \mathrm{Gt} \mathrm{yr}^{-1}$ in 2000 and $(-47 \pm$ 9) $\mathrm{Gt} \mathrm{yr}^{-1}$ in 2006.

For Basin 10 (ice streams draining into Ross Ice Shelf, including the stagnated Kamb Ice Stream), Rignot et al. (2008) report $(+34 \pm 8) \mathrm{Gt} \mathrm{yr}^{-1}$ which is considerably larger than our estimate of $(+11 \pm 6) \mathrm{Gt} \mathrm{yr}^{-1}$.

For Basin 3 (Enderby Land), a previous GRACE analysis (Chen et al. 2006) inferred a much larger positive trend than our estimate. Close examination of the time-series of monthly changes suggests that the trend is induced by interannual variations (increase in 20022004 followed by a stable behaviour in 2005-2007). Whereas the ERS-1/ERS-2 altimetry analyses cited above show little changes in the region, an analysis of Environmental Satellite (ENVISAT) radar altimeter data over 2003-2007 by Legrésy et al. (2007) shows positive trends (presumably due to an accumulation anomaly) that might be compatible to our GRACE results.

Our estimated pan-Antarctic seasonal signal has its minimum in February, its maximum in October and a peak-to-trough difference of $304 \mathrm{Gt}$. An earlier GRACE analysis by Llubes et al. (2007) qualitatively found similar results. Monthly accumulation variations inferred from atmospheric modelling by Van Lipzig et al. (2002) and Cullather et al. (1998) predict mass variations with a minimum in February, a maximum in August or September, respectively, and a peak-to-trough difference of about $175 \mathrm{Gt}$. Although our results 

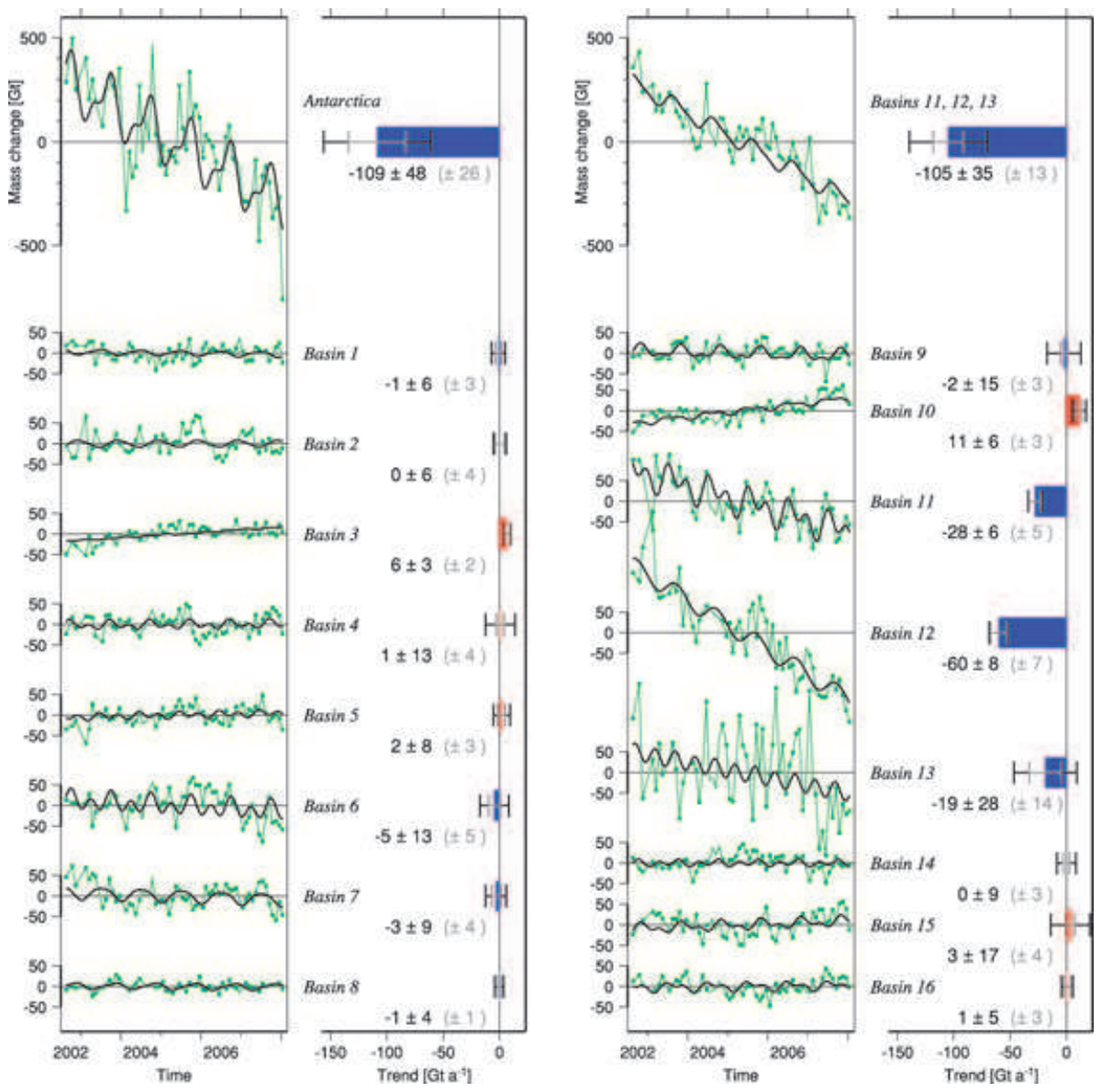

Figure 15. Estimated ice mass changes (Method II) and their trends for entire Antarctica (grounded ice), for the 16 individual drainage basins as shown in Fig. 1, and for the union of Basins 11, 12 and 13. Monthly changes are shown in green (with unregularized months marked by dots), the fitted linear and seasonal models are shown in black. Total rms errors of trends are given in black (numbers and error bars). In addition, errors excluding the GIA error are given in grey.

include GRACE error effects (which likely increase the amplitudes) as well as leakage effects from hydrology and from seasonal Antarctic ice mass variations, the agreement is encouraging.

Finally it is important to compare our results with those of previous GRACE analyses. For the entire grounded ice sheet, Velicogna $\&$ Wahr (2006a) estimate $(-139 \pm 73) \mathrm{Gt} \mathrm{yr}^{-1}$ (ice volume converted to mass with $917 \mathrm{~kg} \mathrm{~m}^{-3}$ density) from UTCSR release-1 monthly solutions over 04/2002-08/2005. Ramillien et al. (2006) estimate $(-40 \pm 36) \mathrm{Gt} \mathrm{yr}^{-1}$ (we quote one-sigma errors) from 10daily 30 -d-solutions by CNES/GRGS over 07/2002-03/2005. By an alternative high-resolution mascon approach using GRACE intersatellite data processing, Luthcke et al. (2007) estimate -81 $\mathrm{Gt} \mathrm{yr}^{-1}$ when GIA is corrected using the IJ05 model. For the Amundsen Sea Sector of West Antarctica (corresponding to our Basin 12 and part

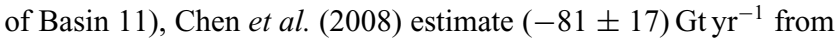
UTCSR release- 4 monthly solutions over $01 / 2003-09 / 2006$ by their approach of fitting a predefined pattern in the spatial domain. By a similar yet somewhat more sophisticated approach, Sasgen et al. (2007) estimate $(-69 \pm 4) \mathrm{Gt} \mathrm{yr}^{-1}$ from the joint analysis of four series of monthly solutions.

In view of the assessed errors, there is no fundamental contradiction between our estimates and those previously determined. Nevertheless, the differences are so large that they motivate further discussion. One cause are different actual ice mass changes related to the different time intervals. However, they are not the only and probably not the main cause. Instead, differences in the methods of GRACE data analysis with their different inherent errors have to be considered as a major cause. These methods (except Luthcke et al. 2007) include the GRACE processing to produce the monthly gravity field solutions and the analysis of these solutions (including geophysical corrections) for mass change inferences. Concerning this last step, we may raise the following points based on our error investigations of Section 4.

The GIA correction applied by Velicogna \& Wahr (2006a) does not rely on a particular model (like IJ05 in our and in most other studies) but uses a mean value of corrections obtained from different models. Since this value is larger than the corrections from the IJ05 model, there likely arises a negative offset between the estimates by Velicogna \& Wahr (2006a) and those of other studies.

The analyses also differ by their account for leakage effects by Antarctic ice mass variations (leakage from inside for an integration over entire Antarctica). Velicogna \& Wahr (2006a) use a rescaling analogous to our approach with Method II but with the relatively large value of $0.61^{-1}=1.61$. In view of our investigations in Section 4.2.1, it is not clear how reliably this rescaling mitigates leakage from inside. Ramillien et al. (2006) use the original regional integration approach based on the regularized $30 \mathrm{~d}$ solutions by CNES/GRGS. They apply no additional filtering, but the spectral truncation and regularization inherent in these solutions corresponds to a particular filter (e.g. Kusche 2007). 
Hence, leakage effects do arise, and they might damp the total mass changes.

The adopted region boundaries also differ. Velicogna \& Wahr (2006a) include the ice shelves into their integration region. Although mass changes of floating ice do not affect gravity due to hydrostasy, this choice makes the results more vulnerable to errors in the GIA models (which show large signals in the Ross and Filchner Ronne Ice Shelves) and to residual tidal effects. The analyses by Chen et al. $(2008,2006)$ allow for mass changes only in restricted areas. By not estimating mass changes in most parts of Antarctica, it can not be easily extrapolated to a pan-Antarctic scale. Similar remarks hold for the study by Sasgen et al. (2007), although here the allowed-for patterns of mass change are not restricted in area but still in their number (three in total).

\section{CONCLUSIONS AND OUTLOOK}

We have examined the estimation of mass changes from GRACE monthly solutions. Among other things, we assessed leakage induced by the non-uniform weighting within Antarctica using a range of ice mass variation scenarios. Restricting such an assessment to a single (e.g. uniform) scenario may mislead to corrections that might even aggravate leakage effects. We further showed that by error correlations of the GRACE monthly solutions, the GRACE error effects on Antarctic mass changes are about two times larger than predicted by standard error models that do not include correlations. Presumable temporal correlations of GRACE errors are an additional error source. These insights are relevant, also beyond our particular application to the Antarctic ice sheet.

Beyond the concrete mass change estimates reported in the previous section, our analyses indicate directions of methodological improvements. Some of them may build on the current monthly solution releases. The incorporation of a priori information may be further enhanced based on the approach of predefined patterns. In fact, the upscaling applied with Method II is an attempt to replace the uncertain degree-1 and -2 information by higher-degree information, and, thus, heuristically exploits known couplings between low and high degrees. The predefined patterns approach is a more solid incorporation of such a priori information. More realistic patterns could be derived from altimetry, SMB modelling, flow velocity fields and other sources. Applying more realistic error models of the GRACE solutions is another obvious improvement. Note, however, that presently, even the complete formal covariance matrix does not fully describe the error structures (Horwath 2007; Schrama et al. 2007). Modelling and parametrization problems in the GRACE processing are reasons for this discrepancy (e.g. Wiehl \& Dietrich 2005; Seo et al. 2008).

Concerning necessary developments in the GRACE processing, improvements of the background models are remaining an important task. The functional models linking GRACE observations to gravity field variations are another issue. The traditional approach of numerical dynamic orbit analysis (Reigber et al. 2005) has attractive alternatives tailored to the new satellite gravity missions (Rowlands et al. 2002; Mayer-Grr et al. 2005; Ilk et al. 2008). As yet another field of development, the parametrization of spatio-temporal gravity field or mass variations has to seek proper compromises between detectability and geophysical relevance of the parametrized variations (Wiehl \& Dietrich 2005; Horwath et al. 2007a). From the geophysical viewpoint, a regionalized parametrization seems attractive. The mascon approach (Luthcke et al. 2006, 2007) is one example. Indeed, if GRACE shall observe mass transports in the Earth system, it seems more direct to parametrize the mass changes than to go across the intermediate product of global gravity field models. Finally, only by its consistent combination with complementary observations and models will GRACE fulfill its role as one 'sensor' in a consistent Global Geodetic Observing System (Drewes 2005). Preferably, the synthesis of different techniques should occur at an early stage (for example, affecting the GRACE processing) to consistently exploit complementarities.

\section{ACKNOWLEDGMENTS}

Many colleagues inspired and supported this work by interesting discussions and by providing data sets for this paper or for background analyses contained in Horwath (2007). We especially thank Benoît Legrésy at Laboratoire d'Études en Gophysique et Ocanographie Spatiales (LEGOS), Toulouse, for radar altimetry results, Erik Ivins at Jet Propulsion Laboratory (JPL), Pasadena, for the GIA model, Petra Döll at Johann Wolfgang Goethe-Universität Frankfurt and Svetozar Petrovic at GFZ Potsdam for global hydrology models, Roland Schmidt at GFZ Potsdam for results of a full GRACE error covariance matrix propagation and Hans Oerter at Alfred-WegenerInstitut für Polar- und Meeresforschung Bremerhaven for in situ accumulation data. We gratefully acknowledge comments by two anonymous reviewers and editorial help by Erik Ivins, which lead to great improvements of the original manuscript. This work was supported by the German Research Foundation (DFG) under grants Di 473/13, Di 473/17 (within the Geotechnologien Programme) and Di 473/31 (within the Priority Programme SPP1257 'Mass Transports and Mass Distribution in the Earth System').

\section{REFERENCES}

Bentley, C.R., 2004. Mass balance of the Antarctic ice sheet: observational aspects, in Mass Balance of the Cryosphere: Observations and Modelling of Contemporary and Future Changes, pp. 459-490, eds Bamber, J.L. \& Payne, A.J., Cambridge University Press, Cambridge, UK.

Chen, J.L., Wilson, C.R., Blankenship, D.D. \& Tapley, B.D., 2006. Antarctic mass rates from GRACE, Geophys. Res. Lett., 33, L11502, doi:10.1029/2006GL026369.

Chen, J.L., Wilson, C.R., Famiglietti, J.S. \& Rodell, M., 2007. Attenuation effect on seasonal basin-scale water storage changes from GRACE time-variable gravity, J. Geod., 81(4), 237-245, doi:10.1007/s00190006-0104-2.

Chen, J.L., Wilson, C.R., Tapley, B.D., Blankenship, B. \& Young, D., 2008. Antarctic regional ice loss rates from GRACE, Earth planet. Sci. Lett., 266, 140-148, doi:10.1016/j.epsl.2007.10.057.

Cullather, R.I., Bromwich, D.H. \& van Woert, M.L., 1998. Spatial and temporal variability of Antarctic precipitation from atmospheric methods, J. Clim., 11, 334-367.

Davis, C.H., Li, Y., McConnell, J.R., Frey, M.M. \& Hanna, E., 2005. Snowfall-driven growth in East Antarctic Ice Sheet mitigates recent sealevel rise, Science, 308(5730), 1898-1901, doi:10.1126/science.1110662.

Döll, P., Kaspar, F. \& Lehner, B., 2003. A global hydrological model for deriving water availability indicators: model tuning and validation, $J$. Hydrol., 270(1-2), 105-134, doi:10.1016/S0022-1694(02)00283-4.

Drewes, H., 2005. Science Rationale of the Global Geodetic Observing System (GGOS), in Dynamic Planet: Monitoring and Understanding a Dynamic Planet with Geodetic and Oceanographic Tools, IAG Symposia,Vol. 130, pp. 703-710, Springer, Berlin.

Farrell, W.E., 1972. Deformation of the Earth by Surface Load, Rev. Geophys. Space Phys., 10(3), 761-797.

Fenoglio-Marc, L., Kusche, J. \& Becker, M., 2006. Mass variation in the Mediterranean Sea from GRACE and its validation by altimetry, steric and hydrologic fields, Geophys. Res. Lett., 33(19), L19606, doi:10.1029/2006GL026851. 
GFZ, 2007. Release notes for GFZ Potsdam GRACE L-2 products - version RL04, available at http://isdc.gfz-potsdam.de.

Han, S.-C., Shum, C.K. \& Matsumoto, K., 2005. GRACE observations of $\mathrm{M}_{2}$ and $\mathrm{S}_{2}$ ocean tides underneath the Filchner-Ronne and Larsen ice shelves, Antarctica, Geophys. Res. Lett., 32, L20311, doi:10.1029/2005GL024296.

Heiskanen, W.A. \& Moritz, H., 1967. Physical Geodesy, W.H. Freeman and Co., San Francisco.

Helsen, M.M., van den Broeke, M.R., van de Wal, R.S.W., van de Berg, W.J., van Meijgaard, E., Davis, C.H., Li, Y. \& Goodwin, I., 2008. Elevation changes in Antarctica mainly determined by accumulation variability, Science, 320(5883), 1626-1629, doi:10.1126/science.1153894.

Horwath, M., 2007. Determining geophysical mass signals with gravity field missions: an analysis of the current status with regard to Antarctica (in German), PhD thesis, Technische Universität Dresden, Fakultät Forst-, Geo- und Hydrowissenschaften, available at http://nbnresolving.de/urn:nbn:de:bsz:14-ds-1203684703310-42192.

Horwath, M. \& Dietrich, R., 2006. Errors of regional mass variations inferred from GRACE monthly solutions, Geophys. Res. Lett., 33, L07502, doi:10.1029/2005GL025550.

Horwath, M., Dietrich, R., Barthelmes, F., Förste, C. \& Schwintzer, P., 2007a. CHAMP normal equation analyses for assessing sensitivities and parameter correlations, in Proceedings of the Joint CHAMP/GRACE Science Meeting, Potsdam, 2004 July 6-8, available at http://www.gfzpotsdam.de/pb1/JCG/.

Horwath, M., Dietrich, R., Huybrechts, P. \& Linow, S., 2007b. Antarctic ice mass change estimates from GRACE: Results, uncertainties, and the combination with complementary information, in Proceedings of the GRACE Science Team Meeting and DFG SPP1257 Symposium, Potsdam, Germany, 2007 October 15-17, available at http://www.massentransporte.de/index.php?id=gstm07.

Ilk, K.-H., Löcher, A. \& Mayer-Gürr, T., 2008. Do we need new gravity field recovery techniques for the new gravity field satellites?, in $V I$ Hotine-Marussi Symposium on Theoretical and Computational Geodesy, IAG Symposia, Vol. 132, pp. 3-9, doi:10.1007/978-3-540-74584-6_1, Springer, Heidelberg.

Ivins, E.R. \& James, T.J., 2005. Antarctic glacial isostatic adjustment: a new assessment, Antarctic Sci., 17(4), 541-553.

Ivins, E.R. \& James, T.S., 2006. GIA and ice sheet altimetry in Antarctica: how well can we quantify the associated error and its spatial dependence, in Proceedings of the EGU General Assembly, Vienna, 2006 April 3-7, Geophys. Res. Abstr., Vol. 8, pp. EGU06-A-10708, Copernicus Publications, Katlenburg-Lindau, available at http://geophys-res-abstr.net/.

Koch, K.-R., 1999. Parameter Estimation and Hypothesis Testing in Linear Models, 2nd edn, Springer, New York.

Kusche, J., 2007. Approximate decorrelation and non-isotropic smoothing of time-variable GRACE-type gravity field models, J. Geod., 81(11), 733-749, doi:10.1007/s00190-007-0143-3.

Legrésy, B., Rémy, F. \& Blarel, F., 2006. Along track repeat altimetry for ice sheets and continental surface studies, in Proceedings of the Symposium on 15 years of Progress in Radar Altimetry, Venice, Italy, 2006 March 1318, ESA-SP 614, Paper no. 181, ESA Publication Division, Noordwijk, The Netherlands.

Legrésy, B., Ramillien, G., Rémy, F. \& Lemoine, J.-M., 2007. Mass balance of the ice sheets surveyed by space altimetry and gravimetry, in Proceedings of the IUGG XXIV General Assembly, 2007 July 7-13, Perugia, Italy.

Llubes, M., Lemoine, J.-M. \& Rémy, F., 2007. Antarctica seasonal mass variations detected by GRACE, Earth planet. Sci. Lett., 260(1-2), 127136, doi:10.1016/j.epsl.2007.05.022.

Luthcke, S.B. et al., 2006. Recent Greenland ice mass loss by drainage system from satellite gravity observations, Science, 314(5803), 12861289, doi:10.1126/science.1130776.

Luthcke, S.B., Rowlands, D.D., Lemoine, F.G., Zwally, H.J., Hall, D., Arendt, A. \& McCarthy, J.J., 2007. High latitude land ice mass flux from GRACE mascons, in Proceedings of the GRACE Science Team Meeting and DFG SPP1257 Symposium, 2007 October 15-17, Potsdam, Germany, available at http://www.massentransporte.de/index.php?id=gstm07.
Mayer-Grr, T., Ilk, K., Eicker, A. \& Feuchtinger, M., 2005. ITG-CHAMP01: a CHAMP gravity field model from short kinematical arcs of a one-year observation Period, J. Geod., 78(7-8), 462-480, doi:10.1007/s00190004-0413-2.

Milly, P. \& Shmakin, A., 2002. Global modeling of land water and energy balances, part I: the land dynamics (LaD) model, J. Hydrometeorol., 3(3), 283-299.

Monaghan, A.J., Bromwich, D.H. \& Wang, S.-H., 2006. Recent trends in Antarctic snow accumulation from Polar MM5 simulations, Phil. Trans. R. Soc. A, 364(1844), 1683-1708, doi:10.1098/rsta.2006.1795.

Moore, P. \& King, M.A., 2008. Antarctic ice mass balance estimates from GRACE: tidal aliasing effects, J. geophys. Res., 113(F02005), doi:10.1029/2007JF000871.

Peltier, W.R., 1998. Postglacial variations in the level of the sea: Implications for climate dynamics and solid-earth geophysics, Rev. Geophys., 36(4), 603-689.

Peltier, W.R., 2004. Global glacial isostasy and the surface of the Ice-Age Earth: the ICE-5G (VM2) Model and GRACE, Ann. Rev. Earth planet. Sci., 32(1), 111-149, doi:10.1146/annurev.earth.32.082503.144359.

Ramillien, G., Cazenave, A. \& Brunau, O., 2004. Global time variations of hydrological signals from GRACE satellite gravimetry, Geophys. J. Int., 158, 813-826.

Ramillien, G., Frappart, F., Cazenave, A. \& Güntner, A., 2005. Time variations of land water storage from an inversion of 2 years of GRACE geoids, Earth planet. Sci. Lett., 235, 283-301, doi:10.1016/j.eps1.2005.04.005.

Ramillien, G., Lombard, A., Cazenave, A., Ivins, E.R., Llubes, M., Rémy, F. \& Biancale, R., 2006. Interannual variations of the mass balance of the Antarctica and Greenland ice sheets from GRACE, Global Planet. Change, 53, 198-208, doi:10.1016/j.gloplacha.2006.06.003.

Reigber, C., Schmidt, R., Flechtner, F., König, R., Meyer, U., Neumayer, K., Schwintzer, P. \& Zhu, S., 2005. An Earth gravity field model complete to degree and order 150 from GRACE: EIGEN-GRACE02S, J. Geodyn., 39, 1-10, doi:10.1016/j.jog.2004.07.001

Rignot, E., Bamber, J., van den Broeke, M.R., Davis, C., Li, Y., van de Berg, W.J. \& van Meijgaard, E., 2008. Recent Antarctic ice mass loss from radar interferometry and regional climate modelling, Nat. Geosci., 1, 106-110, doi:10.1038/ngeo102.

Rodell, M. et al., 2004. The global land data assimilation system, Bull. Am. Meteorol. Soc., 85(3), 381-394, doi:10.1175/BAMS-85-3-381.

Rowlands, D., Ray, R., Chinn, D. \& Lemoine, F., 2002. Short-arc analysis of intersatellite tracking data in a gravity mapping mission, J. Geod., 76, 307-316, doi: 10.1007/s00190-002-0255-8.

Sasgen, I., Martinec, Z. \& Fleming, K., 2007. Regional ice-mass changes and glacial-isostatic adjustment in Antarctica from GRACE, Earth planet. Sci. Lett., 264, 391-401, doi:10.1016/j.eps1.2007.09.029.

Schmidt, R. et al., 2006. GRACE observations of changes in continental water storage, Global Planet. Change, 50(1-2), 112-126, doi:10.1016/j.placha.2004.11.018.

Schrama, E.J.O., Wouters, B. \& Lavallée, D.A., 2007. Signal and noise in Gravity Recovery and Climate Experiment (GRACE) observed surface mass variations, J. geophys. Res., 112, B08407, doi: $10.1029 / 2006 J B 004882$.

Seo, K.-W., Wilson, C.R., Famiglietti, J.S., Chen, J.L. \& Rodell, M., 2006. Terrestrial water mass load changes from Gravity Recovery and Climate Experiment (GRACE), Water Resour. Res., 42, W05417, doi:10.1029/2005WR004255.

Seo, K.-W., Wilson, C.R., Chen, J. \& Waliser, D.E., 2008. GRACE's spatial aliasing error, Geophys. J. Int., 172, 41-48, doi:10.1111/j.1365246X.2007.03611.x.

Solomon, S., Qin, D., Manning, M., Chen, Z., Marquis, M., Averyt, K.B., Tignor, M. \& Miller, H.L., 2007. Climate Change 2007: The Physical Basis. Contribution of Working Group I to the Fourth Assessment Report of the Intergovernmental Panel on Climate Change, Cambridge University Press, Cambridge.

Swenson, S. \& Wahr, J., 2002. Methods for inferring regional surface-mass anomalies from Gravity Recovery and Climate Experiment (GRACE) measurements of time-variable gravity, J. geophys. Res., 107(B9), 2193, doi:10.1029/2001B000576. 
Swenson, S. \& Wahr, J., 2006. Post-processing removal of correlated errors in GRACE data, Geophys. Res. Lett., 33, L08402, doi:10.1029/2005GL025285.

Tapley, B., Bettadpur, S., Ries, J., Thompson, P. \& Watkins, M., 2004. GRACE Measurements of Mass Variability in the Earth System, Science, 305(5683), 503-505, doi:10.1126/science.1099192.

Van Lipzig, N., van Meijgaard, E. \& Oerlemans, J., 2002. The spatial and temporal variability of the surface mass balance in Antarctica: results from a regional atmospheric climate model, Int. J. Climatol., 22, 1197-1217, doi:10.1002/joc. 798 .

Vaníček, P. \& Krakiwsky, E.J., 1986. Geodesy: The Concepts, 2nd edn, North Holland, Amsterdam, New York, Oxford, Tokyo.

Vaughan, D.G., Bamber, J.L., Giovinetto, M., Russell, J. \& Cooper, P.R., 1999. Reassessment of net surface mass balance in Antarctica, J. Clim., 12, 933-946.

Velicogna, I. \& Wahr, J., 2006a. Measurements of time-variable gravity show mass loss in Antarctica, Science, 311(5768), 1754-1756, doi: $10.1126 /$ science. 1123785 .

Velicogna, I. \& Wahr, J., 2006b. Acceleration of Greenland ice mass loss in spring 2004, Nature, 443, 329-331, doi:10.1038/nature05168.

Wahr, J., Molenaar, M. \& Bryan, F., 1998. Time variablity of the Earth's gravity field: hydrological and oceanic effects and their possible detection using GRACE, J. geophys. Res., 103(B12), 30 205-30 229.

Wahr, J., Swenson, S. \& Velicogna, I., 2007. Some Hydrological and Cryospheric Applications of GRACE, in Proceedings of the GRACE Science Team Meeting and DFG SPP1257 Symposium, 2007 October 15-17, Potsdam, Germany, available at http://www.massentransporte.de/index.php?id=gstm07.

Wiehl, M. \& Dietrich, R., 2005. Time-variable gravity seen by satellite missions: on its sampling and its parametrization, in Earth $\mathrm{Ob}$ servation with CHAMP: Results from Three Years in Orbit, pp. 121126, eds Reigber, C., Lühr, H., Schwintzer, P. \& Wickert, J., Springer, Berlin.

Wiehl, M., Dietrich, R. \& Lehmann, A., 2005. How Baltic Sea water mass variations mask the postglacial rebound signal in CHAMP and GRACE gravity field solutions, in Earth Observation with CHAMP: Results from Three Years in Orbit, pp. 181-186, eds Reigber, C., Lühr, H., Schwintzer, P. \& Wickert, J., Springer, Berlin.

Wingham, D.J., Ridout, A.J., Scharroo, R., Arthern, R.J. \& Shum, C.K., 1998. Antarctic elevation change from 1992 to 1996, Science, 282, 456458.

Wingham, D.J., Shepherd, A., Muir, A. \& Marshall, G.J., 2006. Mass balance of the Antarctic ice sheet, Phil. Trans. R. Soc. A, 364(1844), 1627-1635, doi:10.1098/rsta.2006.1792.
Zwally, H.J., Giovinetto, M.B., Li, J., Cornejo, H.G., Beckley, M.A., Brenner, A.C., Saba, J.L. \& Yi, D., 2005. Mass changes of the Greenland and Antarctic ice sheets and shelves and contributions to sea-level rise: 19922002, J. Glaciol., 51(175), 509-527, doi:10.3189/172756505781829007.

\section{APPENDIX: STOCHASTIC MODEL OF SURFACE MASS BALANCE FLUCTUATIONS}

By relative annual surface mass balance (SMB) fluctuations we mean the deviations of the yearly SMB (accumulation minus ablation) from the long-term mean SMB, divided by this long-term mean. We model the relative annual fluctuations as a homogeneous isotropic stochastic field with a spatial autocorrelation function as shown in Fig. 8b and a pointwise STD of 0.19 (i.e. 19 per cent relative annual fluctuation at every Antarctic position). No temporal correlation from year to year is assumed. This model is established by Horwath (2007), based on statistical analyses of in situ observations. To derive absolute fluctuations we use the long-term mean SMB compilation by Vaughan et al. (1999). Our model is comparable with results on surface height fluctuations by Wingham et al. (1998), though at distances beyond $500 \mathrm{~km}$ our autocorrelation is somewhat larger.

It is straight-forward to derive a respective stochastic model of 5 yr trends induced by SMB fluctuations (as needed in Section 4.2.1) or to derive a stochastic model of monthly SMB fluctuations (as needed in Section 4.3.2). For the latter model, we assume that the yearly variance is evenly distributed over the 12 months.

Based on these models, we generate pseudo-random realizations to perform our statistical assessments. For example, we find that the modelled pan-Antarctic annual fluctuations have a STD that is 3.8 per cent of the mean annual pan-Antarctic SMB. Atmospheric modelling studies by Van Lipzig et al. (2002) and Monaghan et al. (2006) report respective STDs between 4 and 7 per cent. To not underestimate SMB fluctuation effects in our assessment in Section 4.3.2, we scale our results with 1.6 (leading to a 6 per cent relative annual STD) to conform more with those atmospheric modelling results. 